\title{
Coarsening of three-dimensional grains in crystals, or bubbles in dry foams, tends towards a universal, statistically scale-invariant regime
}

\author{
Gilberto L. Thomas* and R. M. C. de Almeida \\ Instituto de Física, Universidade Federal do Rio Grande do Sul, Caixa Postal 15051, 91501-051 Porto Alegre, RS, Brazil \\ F. Graner \\ Spectrométrie Physique, UMR 5588 CNRS, Université Joseph Fourier, 140 rue de la Physique, BP 87, 38402 Saint Martin d'Hères \\ Cedex, France
}

(Received 16 March 2006; published 17 August 2006)

\begin{abstract}
We perform extensive Potts model simulations of three-dimensional dry foam coarsening. Starting with 2.25 million bubbles, we have enough statistics to fulfill the three constraints required for the study of statistical scale invariance: first, enough time for the transient to end and reach the scaling state; then, enough time in the scaling state itself to characterize its properties; and finally, enough bubbles at the end to avoid spurious finite size effects. In the scaling state, we find that the average surface area of the bubbles increases linearly with time. The geometry (bubble shape and size) and topology (number of faces and edges), as well as their correlations, become constant in time. Their distributions agree with the data of the literature. We present an analytical model (universal, up to parameters extracted from the simulations) for a disordered foam minimizing its free energy, which agrees with the simulations. We discuss the limitations of the simulations and of the model.
\end{abstract}

DOI: 10.1103/PhysRevE.74.021407

PACS number(s): 82.70.Rr, 81.10.Aj, 81.15.Aa, 83.80.Iz

\section{INTRODUCTION}

The coarsening of dry foams is a model of grain growth in crystals [1,2], and is a fascinating process in itself [3-5]. The mechanical equilibration of the soap film separating the bubbles in a foam requires that three walls meet at each bubble edge, forming equal angles of $120^{\circ}$, and four edges meet at each vertex, forming symmetrical solid angles. In a foam made of bubbles with different numbers of faces, this geometry is possible due to the fast rearrangement of edges and vertices that produce differences in gas pressure of neighboring bubbles. These pressure differences, on their turn, cause the bending of walls and edges such that bubbles in a foam resemble polyhedra with curved faces. The internal angles between faces of a polyhedron with a large number of faces $f$ are, in average, larger than $120^{\circ}$, and hence a large $f$ bubble in a foam will have its walls rearranged to present concave curvatures reducing the angles between faces.

In a much slower time scale when compared to walls and edges movement, the pressure difference between neighboring bubbles drives gas diffusion across the curved walls, such that bubbles with a large number $f$ of faces, concave walls, and filled with gas at a lower pressure, grow at the expense of small $f$ bubbles, which are at a higher pressure and present convexly curved walls. A small $f$ bubble thus shrinks, loses faces, and shrinks even more quickly, until it disappears. At that instant, its neighbors lose a face: those which were expanding start expanding more slowly, and those which were steady begin to shrink. Thus there is a constant decrease in the number $N$ of bubbles with the consequent increase in the average bubble size, which goes as $N^{-1}$.

*Electronic address: glt@if.ufrgs.br
In two-dimensional (2D) experiments, the evolution of an individual bubble is simple: the von Neumann law, both linear and exact, states that the growth rate of a $n$-sided bubble is proportional to $6-n[2,4,6]$. The global evolution of the foam is more complicated, but both simulations and theory agree that a statistically "scale invariant" state exists. At long times, the distributions of number of faces (whose average is fixed at 6 by Euler theorem [5]) and of normalized bubble area $[A /\langle A(t)\rangle]$ remain invariant during the coarsening $[4,6,7]$. In this regime, the number of bubbles, the average bubble size, and average area vary, respectively, as $t^{-1}, t^{0.5}$, and $t$, as required by dimensional analysis $[6,8]$.

In three dimensions the situation is much more complicated than in two dimensions [9]. First, there is an additional free parameter: in three dimensions, the average number of faces $\langle f\rangle$ is not determined by the Euler relation between the total number of edges, faces, and vertices in foam [5], and depends on the distribution of the number epf of edges per face: $\langle f\rangle=12 /(6-\langle e p f\rangle)$. Second, the individual growth rate of a 3D bubble found by Mullins [10] and confirmed by numerous others [11-18] is not related to $f$ as simply as in its 2D counterpart, the von Neumann law. In fact, a direct proof of the existence of a 3D scaling state is still lacking.

The only strictly exact 3D theoretical result is a necessary but not sufficient condition. If a scaling state exists, the average bubble size $l$, surface area $s$, and volume $v$ must grow with time with exponents $0.5,1$, and 1.5 , respectively. Mullins has demonstrated it in detail for both foams and grains in his 1986 paper [19]. Glazier later showed that it results in fact from a short dimensional argument [8].

Since Glazier's pioneering paper [20], 3D experiments [8,21-24] and simulations $[12,15,20,25,26]$ are making quick progress; for reviews, see Refs. $[8,15]$. There is a growing consensus that, starting from different disordered initial conditions, the distributions of normalized bubble volumes 
$v /\langle v(t)\rangle$ and numbers of faces $f$ relax towards an asymptotic state $[15,25,26]$.

However, these studies do not yet manipulate enough bubbles to satisfy the following three requirements: (i) enough time for the transient to end and reach the scaling state, (ii) then enough time to characterize the properties of the scaling state itself, and finally (iii) enough bubbles at the end to avoid spurious finite size effects. The largest simulation up to now [12] starts with 1000 bubbles, and ends with 100. It indicates that after a transient the grain distribution apparently reaches a scaling state with $\langle f\rangle=13.5$, decreasing from 14 in the initial condition. The evolution of the average bubble size is also compatible with the expected exponent 0.5 . However, a direct observation of the scaling state is still missing.

The plan of the present paper is as follows. We first present the Potts model, with which we simulate millions of coarsening bubbles (Sec. II). We then collect a catalog of our measurements: geometry (bubble shape and size) and topology (bubble number of faces and edges), as well as their correlations, at different times during the coarsening (Sec. III). We present an analytical model for a disordered foam, based on free energy minimization, with only one free parameter, which agrees with the simulations (Sec. IV). Finally, we discuss the limitations of the Potts simulations and analytical model (Sec. V).

\section{METHODS OF POTTS MODEL SIMULATIONS}

\section{A. Potts model parameters}

We use the Potts model [27,28] (reviewed in Ref. [29]) to simulate the coarsening of a foam or of grains in crystals, as in Refs. $[6,20,25]$. In what follows, we do not distinguish between grains (which is, strictly speaking, what the Potts model simulates) and bubbles. For discussions of some common points and differences, see, for instance, Ref. [15].

The foam consists of $N$ bubbles, described as follows. We consider a 3D cubic lattice with periodic boundary conditions. Each lattice site in this 3D network is represented by an integer $i=1, \ldots, p$, where $p=L_{x} \times L_{y} \times L_{z}$ is the size of the lattice. Each site also has a label, an integer number $S_{i}$ $=1, \ldots, N$. A bubble is defined as a connected set of equally labeled sites, with their label $S$ being used to refer to that bubble. The lattice is then tiled by $N$ bubbles.

The energy of the foam is proportional to the area of the walls between neighboring bubbles; the coefficient of proportionality is the surface energy $J[5]$. On a lattice, the expression for this energy (or, in fact, a Hamiltonian) takes a discrete form

$$
E=\frac{1}{2} \sum_{i} \sum_{\langle j\rangle_{i}} J\left[1-\delta\left(S_{i}-S_{j}\right)\right],
$$

where $\langle j\rangle_{i}$ indicates the sum over every site $j$ that is neighbor to site $i$, the factor $1 / 2$ avoids counting twice each bubble wall, and $\delta$ is the Kronecker symbol: $\delta\left(S_{i}-S_{j}\right)=1$ if $S_{i}=S_{j}$ (sites belonging to the same bubble, thus no energy cost) and $\delta\left(S_{i}-S_{j}\right)=0$ if $S_{i} \neq S_{j}$ (different bubbles, thus an energy cost $J$ ). In what follows, we take $J=1$, without loss of generality,

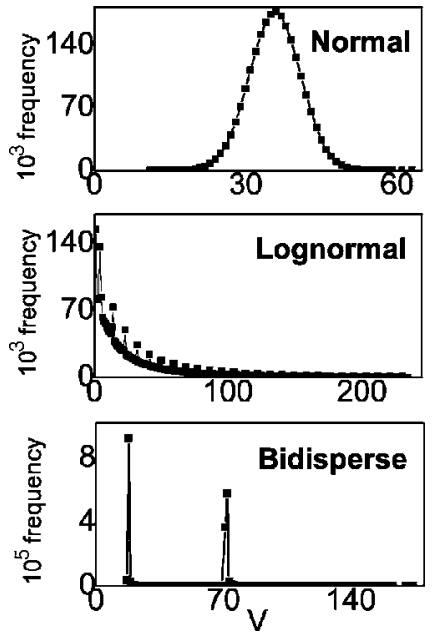

FIG. 1. Volume distributions used as initial conditions after relaxing the system for 300 MCSs using target volume energy term. Observe that this relaxation slightly broadens the bidisperse initial condition.

and we consider first, second, and third neighbors (summing up 26 neighbors) to reduce lattice anisotropy effects [30].

To let the foam evolve, at each time step we randomly choose a site $i$. Among its six first neighbors, we randomly select a site $j$. If $j$ belongs to a different bubble $\left(S_{i} \neq S_{j}\right)$, we move the bubble wall by changing the value $S_{i}$ to $S_{j}$ whenever this represents a decrease in energy. When energy remains the same we change the value $S_{i}$ to $S_{j}$ with $50 \%$ of probability. Finally, when energy would increase, we leave the configuration as is. One Monte Carlo step (MCS) is defined as $p$ random choices (where $p$ is the number of lattice sites); thus, in average, each lattice site is visited at each MCS. Hereafter, for simplicity, we call "time" the number of MCSs.

This evolution procedure simulates the coarsening due to curvature-driven wall movement. Once a set of lattice site is connected, it remains connected. We thus only need to ensure that all bubbles are connected at $t=0$. Initial conditions are produced according to a prescribed distribution of bubble volumes, as follows.

\section{B. Initial conditions}

To avoid unnecessary long transients [31], we use three simple initial volume distributions, as shown in Fig. 1. To obtain the froth initial configuration we first randomly choose $N_{0}$ sites on the cubic lattice. To each of these chosen sites we randomly assign a target volume $v^{\text {target }}$ taken from a given, well defined target volume distribution. We enforce each bubble volume by temporarily adding to Eq. (1) a volume constraint energy term: $E_{\text {target }}=\sum_{i=1}^{N_{0}}\left(v_{i}-v_{i}^{\text {target }}\right)^{2}$; we then let this system evolve as described above for 300 MCSs to obtain the desired initial state: $N_{0}$ bubbles filling all lattice with a predefined volume distribution. The three target volume distributions are listed in what follows:

(i) Normal: initially, bubbles have an average volume of 36 voxels, with a standard deviation of 5 voxels. There are 


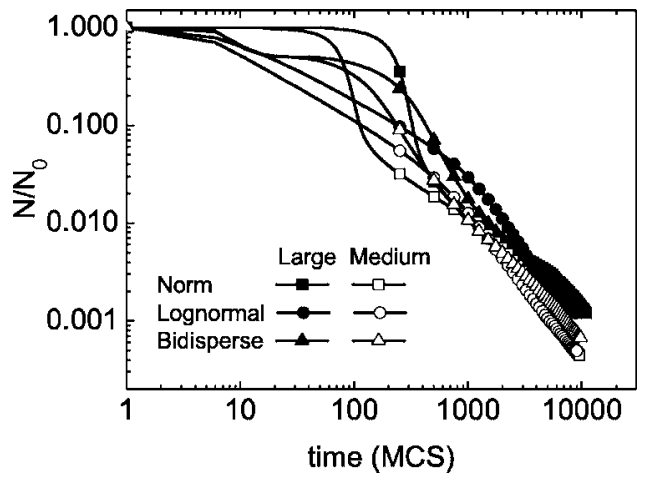

FIG. 2. Relative number of bubbles $N(t) / N_{0}$ as function of time in log-log plot for normal, lognormal, and bidisperse initial conditions (see text), for large and medium size systems.

\section{$2.25 \times 10^{6}$ bubbles on a $450 \times 450 \times 400$ voxels lattice.}

(ii) Log normal: in order to produce an initial state as close as possible to the scale invariant regime, we measure the volume distribution of the preceding normal distribution after 5000 MCSs of coarsening, by building a histogram ranging from 0.0 to 6.5 average volume, using 260 bins. We rescale this distribution to obtain $2.25 \times 10^{6}$ bubbles on a $450 \times 450 \times 400$ voxels lattice, such that the rescaled volume average is 36 pixels. We thus obtain an approximately lognormal distribution peaked at 24 voxels with a standard deviation in the corresponding normal distribution in $\log (v)$ of 1.8 voxels. The periodic peaks in the rescaled volume distribution (see Fig. 1) are numerical artifacts of this procedure. Although these peaks represent a deviation from a pure lognormal distribution, the use of this initial condition shortened the transient, as we show in what follows.

(iii) Bidisperse: initially, half the bubbles have 18 voxels, the other half have 72 voxels. There are $2.025 \times 10^{6}$ bubbles on a $450 \times 450 \times 450$ voxels lattice.

After building one of these initial conditions, the additional volume constraint is switched off and the foam coarsens freely. All runs take typically six weeks on a nonexclusively-dedicated AMD Athlon MP 2400+ computer, to evolve from the order of 2 million down to about 1000 bubbles. To investigate the effects of system size, we also perform medium size simulations with initially $2 \times 10^{5}$ bubbles, with the same three initial volume distributions.

Figure 2 presents the evolution of $N(t) / N_{0}$ for different initial conditions and system sizes, where different transients may be observed. To test whether these systems attain a scaling state, we plot in Fig. 3 the evolution of $\langle v\rangle^{2 / 3},\langle s\rangle$, and $\langle l\rangle^{2}$, where $v, s$, and $l$ are, respectively, volume, surface area, and edge length of the bubbles. By Mullins results [10], these quantities are expected to depend linearly on time in the scaling regime. The plots in the left column present $\langle s\rangle$ versus time for two different system sizes for the three different initial conditions, and in the right column, we compare the effect of initial conditions for large systems by monitoring different quantities.

Both Figs. 2 and 3 show that the relaxation towards the scaling regime is very long; the initial number of bubbles must then be large enough to guarantee that when the scaling regime is attained there are still enough bubbles to yield

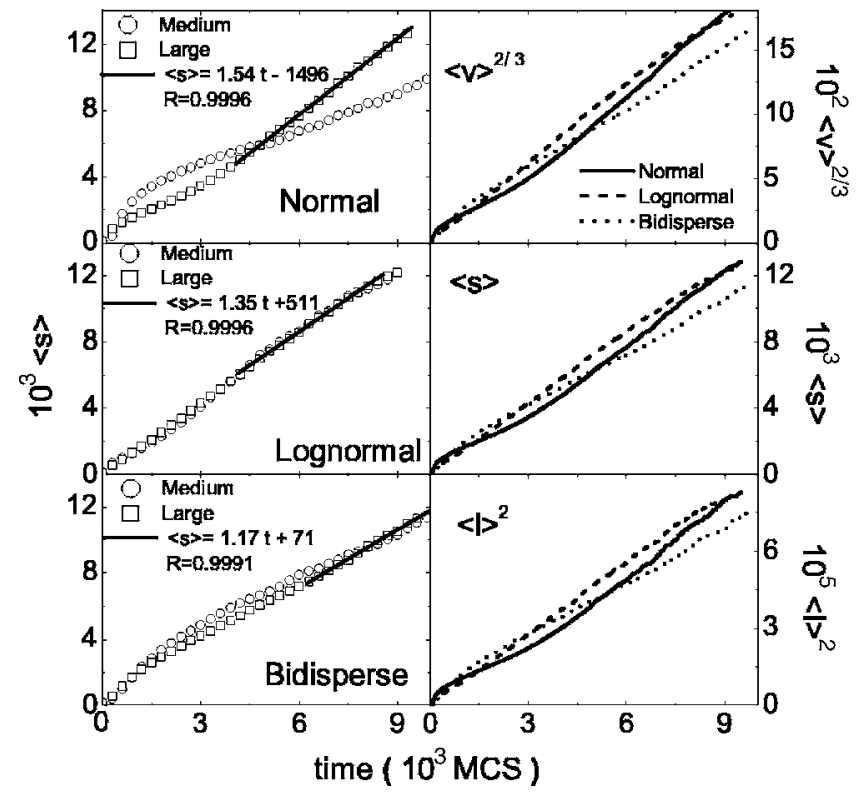

FIG. 3. Left column: Evolution of the average surface area $\langle s\rangle$ for different initial conditions and system sizes, together with linear fits. In the legends the linear fit and its $R$-square value. Right column: Evolution of $\langle v\rangle^{2 / 3},\langle s\rangle$, and $\langle l\rangle^{2}$ for large systems with different initial conditions.

good statistics and to allow a reliable coarsening exponent measurement. We thus present below only large simulations, with $N_{0}$ of the order of $2 \times 10^{6}$ bubbles. They also indicate that the systems tend towards a unique scaling state, although initial conditions with normal volume distributions seem to present longer transients.

\section{Geometrical measurements}

Geometrical quantities are defined as and measured by assigning a lattice site to an elementary cube. The volume of a bubble is the number of cubes it contains. Its surface area is the number of cube faces shared by cubes belonging to two different bubbles. Finally, its edge length is the number of edges that are shared by four cubes belonging to at least three different bubbles.

As is well known [32] in simulations or experiments using pixellized images, geometrical measurements (curvatures, lengths, areas, and in a lesser extent volumes) deviate from the Euclidean measure. As an example, consider the distance $d$ between the site located at the origin of a Cartesian reference frame, $(0,0,0)$, and another site located at $(x, y, z)$. The Euclidean distance is $d_{E}=\sqrt{\left(x^{2}+y^{2}+z^{2}\right)}$, while following the recipe given above $d_{R}=x+y+z$. An alternative way of measuring distances and surfaces in a threedimensional lattice would be counting the number of sites belonging to an edge or to a surface such that sites with one or many external neighbors contribute equally to the surface measure. This alternative recipe also differs from the Euclidean measure. For a straight line between the two points $(0,0,0)$ and $(x, y, z)$, this other recipe yields $d_{A}=\max (x, y, z)$. We chose the first definition because it respects the elementary volumes, surfaces, and lengths. 


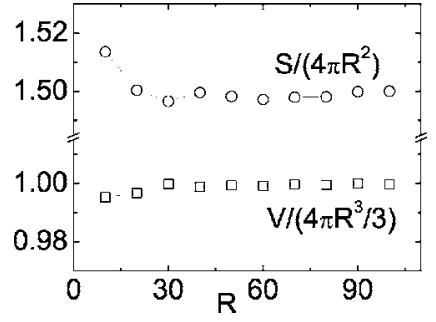

FIG. 4. Measured volume and surface area of a sphere on a cubic lattice, relative to the standard volume and surface. Note the break in the vertical axes.

For instance, we have measured the surface of a sphere on a cubic lattice, for different radii $R$. Since all bubbles are closed, simply connected, three-dimensional bodies, all bubbles have the topology of a sphere. In Fig. 4 we present both $v /\left(4 \pi R^{3} / 3\right)$ and $s /\left(4 \pi R^{2}\right)$. As required to validate our measurements, they tend towards a constant. Since this constant is 1 for the first ratio, but 1.5 for the second one, we need to define a distortion measure suitable for a cubic lattice.

To quantify the bubble deviation from spherical form, Kraynik et al. [33] have introduced the distortion measure $\beta_{i}$ for the $i$ th bubble:

$$
\beta_{i}=\frac{s_{i}}{\left(36 \pi v_{i}^{2}\right)^{1 / 3}},
$$

where $s_{i}$ and $v_{i}$ are, respectively, surface area and volume of the $i$ th bubble. Foam bubbles, besides filling a threedimensional space, must also obey Plateau equilibrium rules: e.g., walls meet at $120^{\circ}$ angles, and walls have a constant mean curvature [5]. In practice, a consequence of Plateau rules in three dimensions (exactly as in two dimensions [34]) is that $\beta$ for all bubbles in a disordered foam is weakly dependent on the number of faces (within 1 or $2 \%$ ). This value is just above the value of regular ones [17], namely 1.097 for the 14-faced Kelvin polyhedra, or 1.095 for the (probably optimal) Weaire-Phelan structure [5].

Similarly, for a cubic lattice, we introduce $\beta_{i}^{c}$ as

$$
\beta_{i}^{c}=\frac{\beta_{i}}{1.5}=\frac{s_{i}}{\left(121.5 \pi v_{i}^{2}\right)^{1 / 3}} .
$$

With this surface measure, bubbles with $\beta^{c}<1$ are possible. These are bubbles that minimize surface energy when defined over a cubic lattice, which is the case of Potts model simulations.

In Fig. 5 we present $\beta_{c}$ for all bubbles at $t=6500$ MCSs for the large, bidisperse run, when the system is deep in the scaling regime and there are still over 2400 cells left, together with the plot of $\beta$ for isotropic Plateau polyhedra (IPP) and convex, isotropic polyhedra (CIP), as obtained in Ref. [13]. IPPs are curved faces bubbles that obey Plateau rules, while the CIP curve is obtained from interpolation for isotropic, convex polyhedra with flat faces. Our simulations results are much more scattered than those obtained by Kraynik et al. [33] in Surface Evolver simulations for ran-

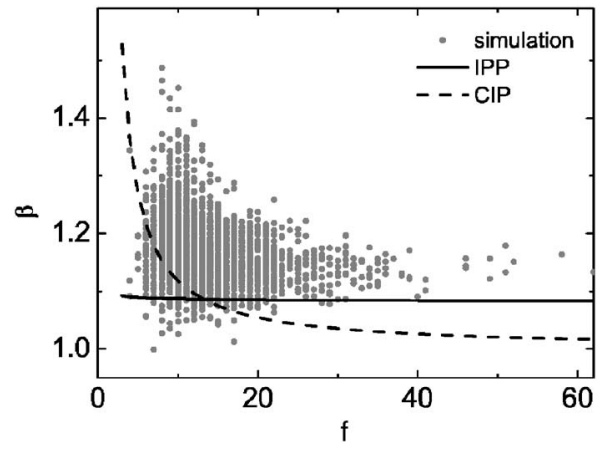

FIG. 5. Cubic lattice surface ratio $\beta_{c}$ for all 2480 bubbles present at $6500 \mathrm{MCSs}$ of the large, bidisperse run, together with calculated values of $\beta$ for isotropic Plateau polyhedra (IPP) and convex isotropic polyhedra (CIP) [13].

dom foams. This probably reflects the fact that we simulate coarsening foams or grains [15], rather than quasistatic structures.

The deviations introduced by the measure recipe are not important when considering quantities given relatively to their average values in the froths. However, they should be taken into account when comparing absolute values obtained from Potts model simulations with analytical or numerical results using continuous representations, such as, for example, the Surface Evolver [35].

A more specific feature of the Potts model is that, to simulate soap froths, one calculates energy terms proportional to the bubble's surface area. One should especially keep in mind that the lattice creates a small but unavoidable anisotropy in the surface energy [30].

Figure 6 checks that in the simulations, measurements of energy (up to third neighbors), and surface (up to first neighbors only) are exactly proportional to each other. In Fig. 6 we present the plot of energy versus surface area for all bubbles in the systems for $t=1000 \mathrm{MCSs}$ and $t=4000 \mathrm{MCSs}$, before and at the scaling regime of an extensive run beginning with lognormal volume distribution. The linear fit is almost perfect and yields $\langle E\rangle=5.94\langle s\rangle+81.87$.

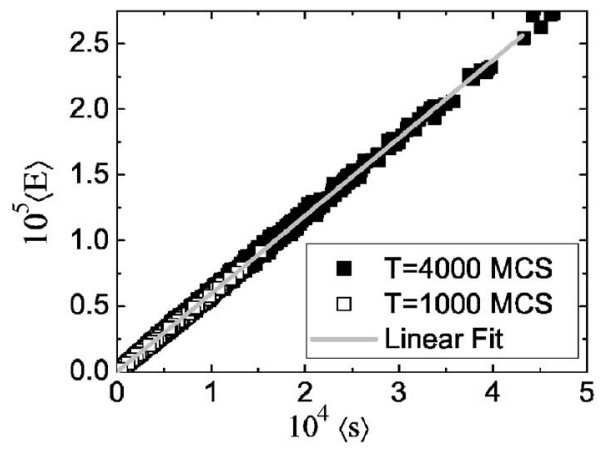

FIG. 6. Average energy versus average surface area of individual bubbles at two specific times of an extensive run beginning with 2.25 million bubbles with lognormal volume distribution. $T$ $=1000$ MCSs (open squares), before the system has attained the scaling regime, and $T=4000 \mathrm{MCSs}$ (solid squares), already in the scaling regime. The solid, gray line is a linear fit (correlation coefficient $R=0.99865),\langle E\rangle=5.94\langle s\rangle+81.87$. 


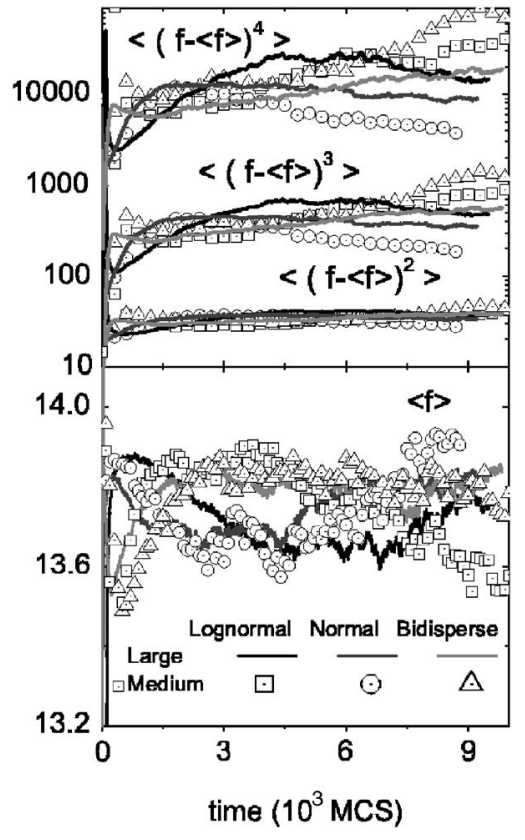

FIG. 7. Evolution of the average number of faces $\langle f\rangle$ (lower plot) and higher order moments (upper plot) for different system sizes (open circles: medium; solid lines: large simulations) and initial conditions (normal, lognormal, and bidisperse). Observe the difference in the scales of the plots, and the vertical log scale in the upper plot.

\section{RESULTS}

\section{A. Evolution of disorder}

We have monitored different physical parameters, characterizing the disorder or dispersion of the system. We begin discussing the evolution of topological measures, represented by the first moments of the distribution in number of faces, as shown in Fig. 7, for different system sizes and different initial conditions, built as explained above. Observe that even the fourth order moment (a quantity very sensitive to details of the distribution) presents reasonably small fluctuations, thanks to the large number of bubbles.

The average number of faces stabilizes around 13.7. The second moment $\mu_{2}=\left\langle(f-\langle f\rangle)^{2}\right\rangle$, however, stabilizes at values higher than reported in the literature; we find $\mu_{2} \sim 37$. At late times the higher moments, $\mu_{3}=\left\langle(f-\langle f\rangle)^{3}\right\rangle$ and $\mu_{4}=\langle(f$ $\left.-\langle f\rangle)^{4}\right\rangle$, typically stabilize at, respectively, 500 and 15000 for the large bidisperse and normal initial condition and 350 and 9000 for the lognormal run.

The evolution of the systems may also be plotted using the time as an implicit parameter. In Fig. 8 we have plotted, as a function of $\mu_{2}$, the average number of faces $\langle f\rangle$ and a conventional measure of volume polydispersity, $\sigma_{R} / R$, defined as

$$
\frac{\sigma_{R}}{R}=\frac{\sqrt{\left\langle(R-\langle R\rangle)^{2}\right\rangle}}{\langle R\rangle}=\frac{\sqrt{\left\langle\left(v^{1 / 3}-\left\langle v^{1 / 3}\right\rangle\right)^{2}\right\rangle}}{\left\langle v^{1 / 3}\right\rangle},
$$

where we have defined for each cell $R=(3 \pi v / 4)^{1 / 3}$. Observe that this polydispersity measure does not use surface or length measures, thus not being affected by the underlying

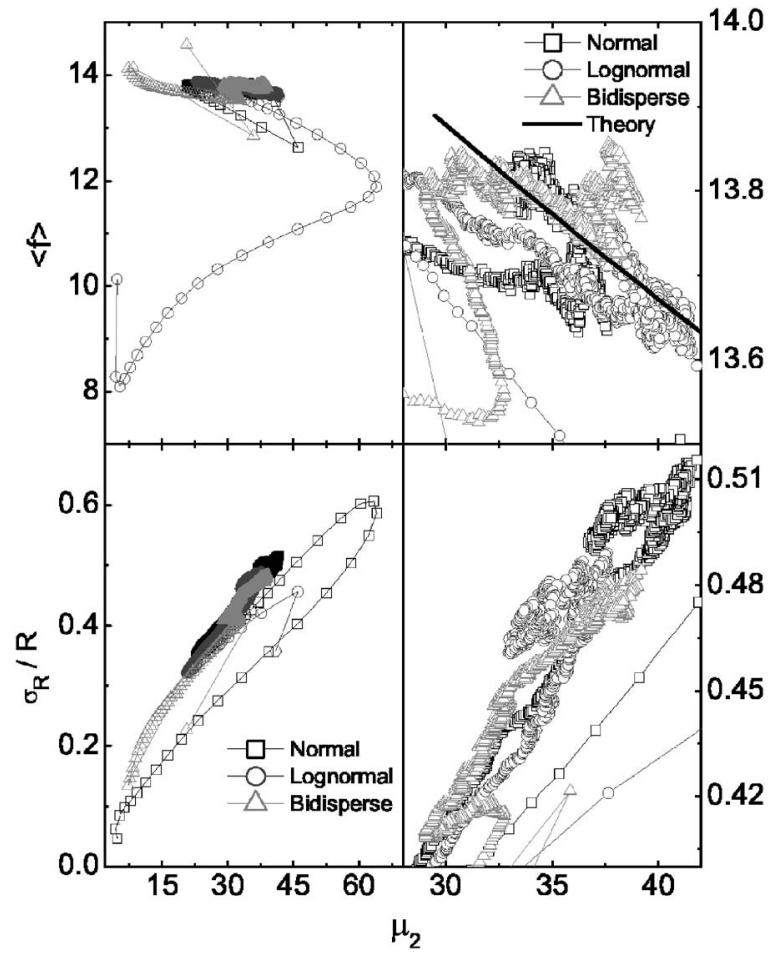

FIG. 8. Trajectories of the large simulations in the $\left(\langle f\rangle, \mu_{2}\right)$ plane and in the $\left(\sigma_{R} / R, \mu_{2}\right)$ plane for different runs (zoomed version in the right columns). Thick, black solid line stands for theoretical predictions as explained in Sec. IV.

lattice. Different initial conditions imply very different initial states, represented by points far apart in the plots, but all tend to a small region centered around $\langle f\rangle=13.7$ or $\sigma_{R} / R=0.48$, and $\mu_{2}=37$. This suggests that there is an asymptotic state independent of initial conditions. The thick, solid line in the upper plot on the right stands for predictions of the model discussed in Sec. IV, that has one free parameter, determined by the average number of faces.

For the lower plots in Fig. 8 we do not present the theoretical predictions, which are typically of the order of 0.6 , about $25 \%$ larger than the values obtained in the simulations. We discuss this point further in what follows. These trajectories show a correlation between $\sigma_{R} / R$ and $\mu_{2}$, indicating higher volume dispersions for higher topological disorders.

Several other dispersion parameters are usually defined as, for example, the ratio $\mu_{2} /\langle f\rangle^{2}$ and polydispersity $p$, defined as suggested in Ref. [17]:

$$
p=\frac{\langle v\rangle^{2 / 3}}{\left\langle v^{2 / 3}\right\rangle}-1 .
$$

Evolution of these three parameters, namely $\sigma_{R} / R, p$, and $\mu_{2} /\langle f\rangle^{2}$, are shown to approach a constant value in Fig. 9, consistently with a scaling regime.

Figure 10 present 2D cuts and 3D images for the different extensive simulations. The 2D cuts (Fig. 10 left and central columns) correspond to a plane parallel to two principal axes of the lattice and are taken at two different times: 1000 MCSs and in the scaling regime, 9000 MCSs. The 3D im- 


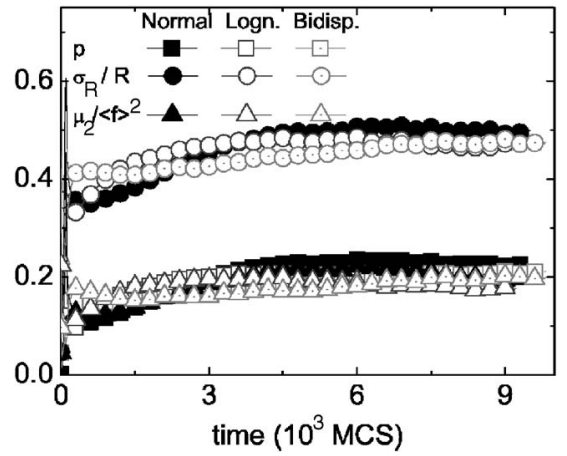

FIG. 9. $\sigma_{R} / R, \mu_{2} /\langle f\rangle^{2}$, and polydispersity $p$ as functions of time for large systems and different initial conditions.

ages are cubes representing $1 / 8$ of the whole system. Observe that even starting with different initial conditions, the final structure of all foams is pretty much the same.

In summary, the simulations presented here seem to indicate that they reach a unique scaling regime, starting from very different initial conditions. The relaxation times are very long and extensive simulations are required to guarantee that when the scaling regime is finally reached, the system still presents a large number of cells to avoid finite size effects and to yield reasonable statistics. In the sections that follow we present the results concerning different distribution functions for the scaling regime.

\section{B. Evolution of geometrical distributions functions}

By geometrical distribution functions we mean distributions in dimensional variables as, for example, volume, sur-

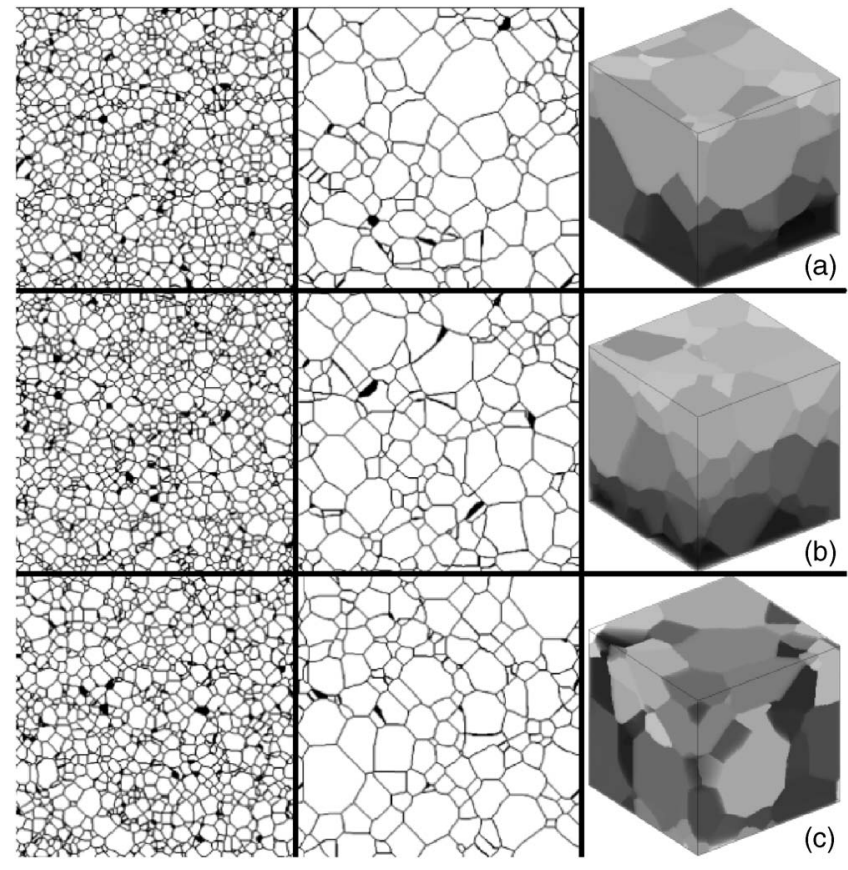

FIG. 10. 2D and 3D extensive simulation images for different initial volume distributions: row (A) normal, row (B) lognormal, and row (C) bidisperse. Left and central columns show 2D cuts for $T=1000$ MCSs and $T=9000 \mathrm{MCSs}$, respectively. Right column shows a portion of the froth at $T=9000 \mathrm{MCSs}$.

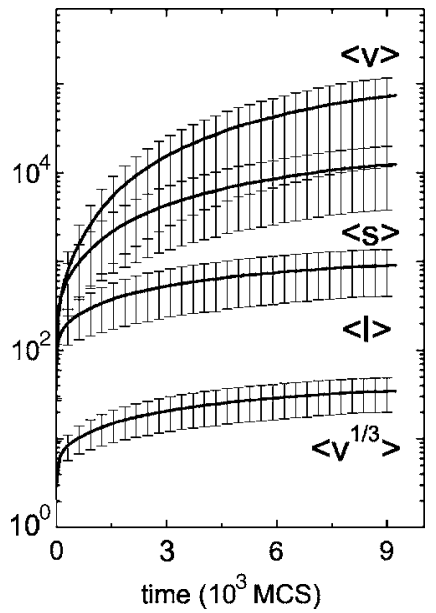

FIG. 11. Evolution of the average values of volume $v, v^{1 / 3}$, surface area $s$, and edge length $l$ for the lognormal large run. The error bars represent standard deviation of a normal distribution in the logarithm of the corresponding variable, or $67 \%$ of the data points assuming the distributions are lognormal.

face area, edge length, and bubble size, here defined as $v^{1 / 3}$. The evolution of the spread of these distributions may be followed in the plot of the average values as a function of time in Fig. 11 with lognormal error bars, given as the standard deviation of a normal distribution for the logarithm of the corresponding variable. The validity of such an assumption is checked in the next section.

We now show the evolution of various distributions. In order to estimate when the scaling state is reached, in Fig. 12 we plot the probability distributions $P$ of nondimensional quantities at different times for three different initial conditions of extensive runs. The nondimensional quantities we followed are the relative volumes $v /\langle v\rangle, v^{1 / 3} /\left\langle v^{1 / 3}\right\rangle$ (more sensitive than $v$ to details in the distributions [12]), surface area $s /\langle s\rangle$, and edge length $l /\langle l\rangle$. All distributions are invariant after $5000 \mathrm{MCSs}$, consistent with a scaling regime. Also in Fig. 12 we present a plot of the scaling regime distribution functions for different initial conditions, which indicates that the same regime can be reached starting from different configurations.

In order to investigate further the scaling state, we have performed an average of distribution functions over different times in the scaling regime: we considered configurations every 25 MCSs in the time interval between 6000 and 9000 MCSs for normal, lognormal, and bidisperse initial conditions. This procedure reduced fluctuations and allowed some conclusions. In Fig. 13 we present the scaling regime averages for the $v /\langle v\rangle$ distribution in linear and log-log (inset) plots, together with the theoretical prediction and a parabolic fit in the log-log plot. The collapse of the simulation data of different runs is perfect, consistent with a unique scaling regime, regardless of initial conditions. The theory agrees with simulations and the fit evidences the lognormal character of the relative volume distribution in the scaling regime. Relative surface area and relative size distribution functions are more sensitive to details in modeling. Their scaling regime averages are shown in Fig. 14, showing a reasonable agreement with theory. 

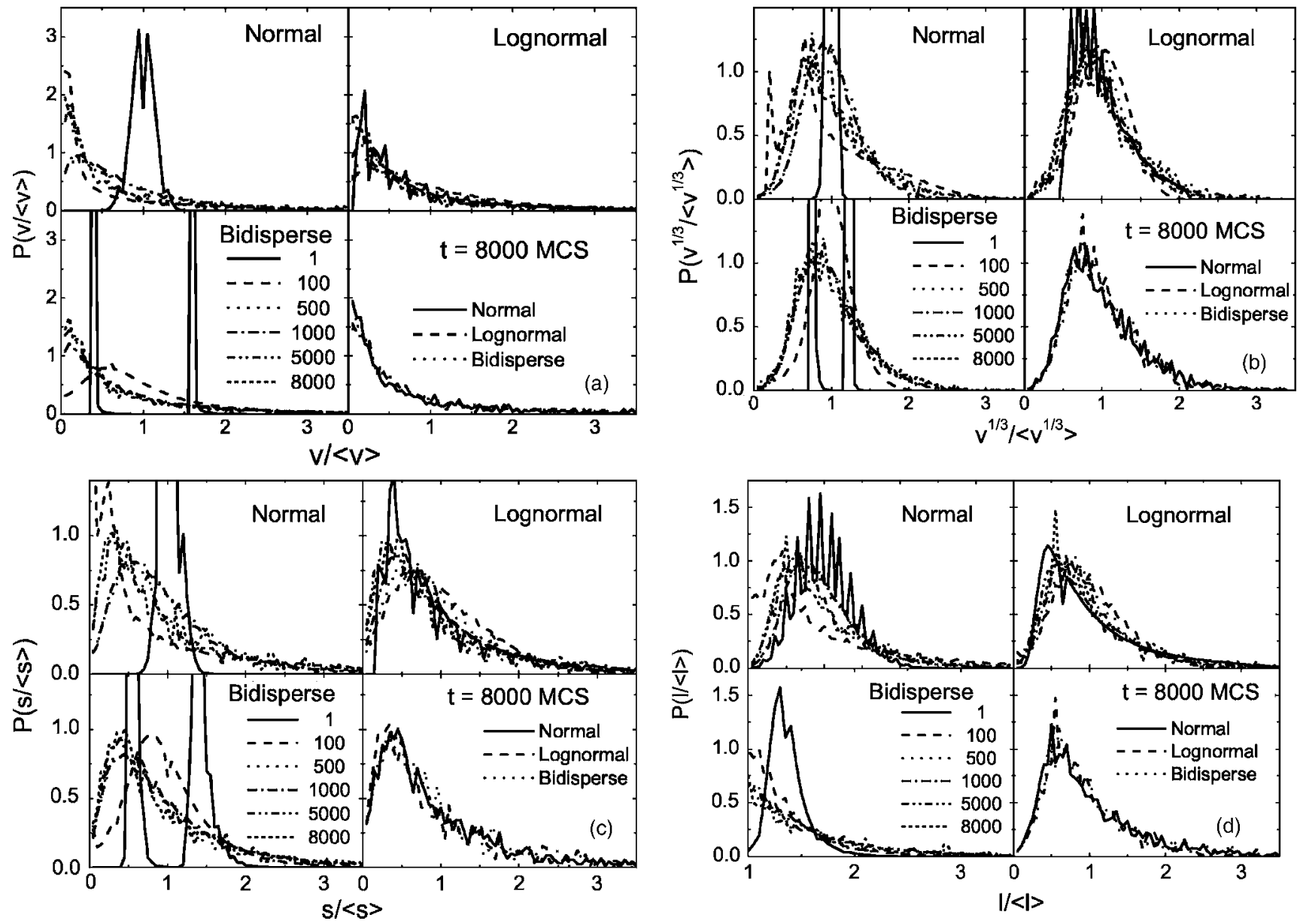

FIG. 12. Successive distributions for different initial conditions of nondimensional (relative) geometrical quantities: (a) volume, (b) equivalent radius $v^{1 / 3}$, (c) surface area, and (d) edge length of the bubbles. Each curve corresponds to a different time, as indicated in the legends. For clarity, each figure is split in 4 , one for each different initial condition and a fourth one containing the scaling regime distributions reached from the three different initial configurations.

We present in Fig. 15 the results for the distribution for $A /\langle A\rangle$ of the relative areas of cells in $2 \mathrm{D}$ cross sections of the three-dimensional simulations, together with experimental

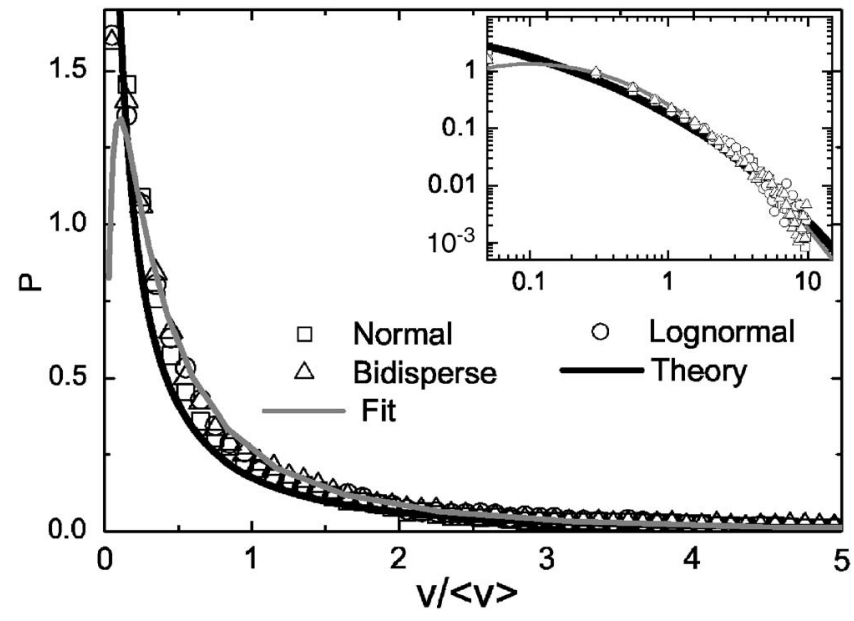

FIG. 13. Scaling regime average of relative volume distribution function for three different initial conditions together with the theoretical prediction and a parabolic fit in log-log plot (inset). and other simulations data as presented in Ref. [12]. To obtain these distributions we have considered, at a given instant, 15 slices in each one of the three principal axes of the lattice, calculated the average area of the cell 2D cross section, and then obtained the distribution in relative areas. In Fig. 15 we present the results for $t=9000$ MCSs for the bidisperse and normal extensive runs, together with the histo-

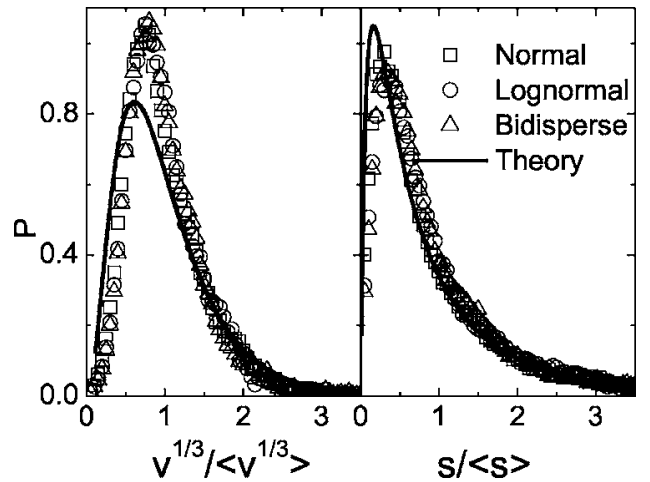

FIG. 14. Scaling regime average of relative size $\left(v^{1 / 3}\right)$ and surface area distribution functions for three different initial conditions together with the theoretical prediction. 


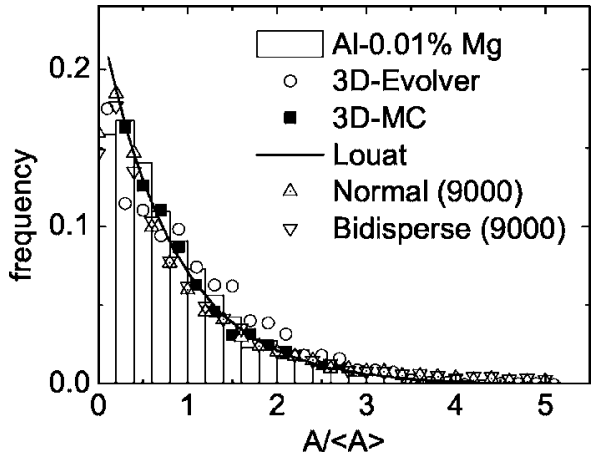

FIG. 15. Distribution of relative area $A /\langle A\rangle$ in $2 \mathrm{D}$ sections of different three-dimensional structures.

gram as obtained in $\mathrm{Al}-0.01 \% \mathrm{Mg}$ as obtained by Fradkov et al. [36], in Potts model 3D simulation by Anderson et al. [25], and in 3D surface evolver simulation by Wakai et al. [12]. The solid line is the transformation of Louat's prediction for these distributions, which gives an exponential.
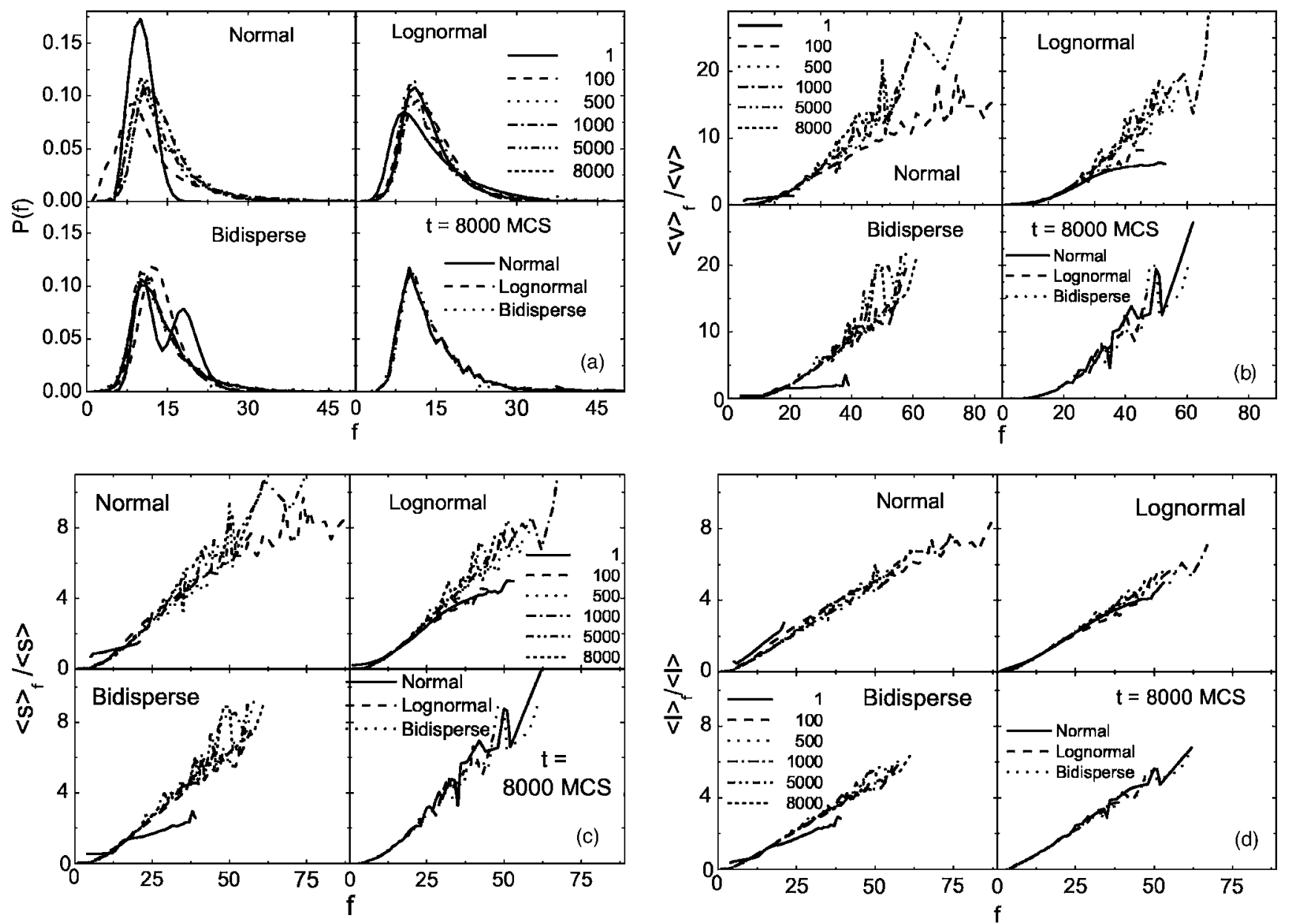

\section{Evolution of topological distributions functions}

Since topological quantities are dimensionless, we plot raw data without rescaling. In Fig. 16 we present the results for $f$-dependent quantities: relative number of $f$-faced cells $P(f)$, average relative volume $\langle v\rangle_{f} /\langle v\rangle$, surface area $\langle s\rangle_{f} /\langle s\rangle$, and edge length $\langle l\rangle_{f} /\langle l\rangle$ of $f$-faced bubbles. Again, we observe that the functions become time independent for late times, in agreement with a scaling regime.

We now address Fig. 17 where we have plotted the scaling averages for the relative surface area and edge length of $f$-faced cells. The edge length is fairly linear in $f$. For a single, cell Euler relation states that $e+2=v+f$, where $e, v$, and $f$ are, respectively, the number of edges, vertices, and faces of the cell. Since $2 e=3 v$, then $e=3 f-6$, that is, the number of edges of the cells depends linearly on the number of faces. The linear relation in the plot indicates then that the average length of a given edge is uniform in the foam. This is consistent with the fact that bubbles with different $f$ share edges.

Faces are also shared by neighboring cells and one should expect that the average surface area per face should not

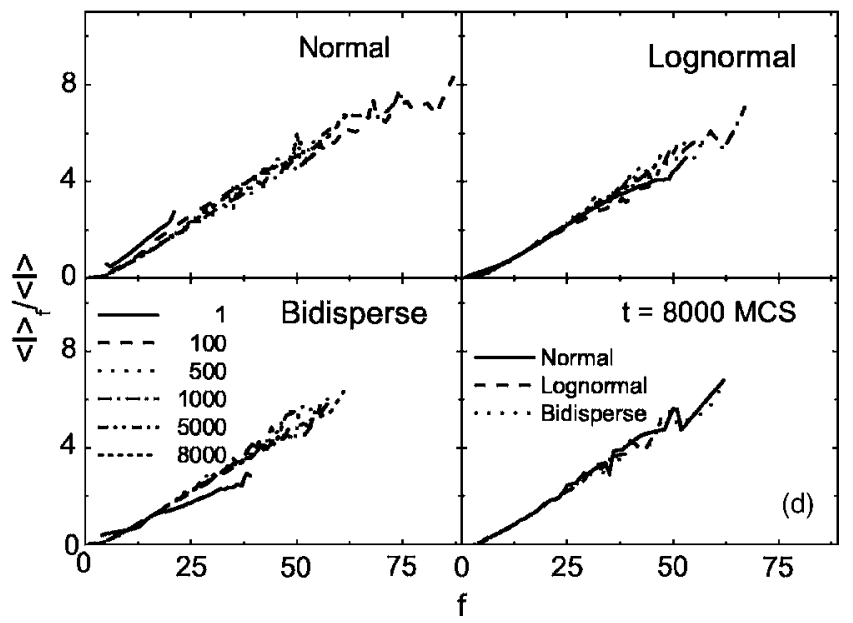

FIG. 16. Successive distributions of $f$-dependent quantities: (a) relative proportion, (b) average volume, (c) average surface area, and (d) average edge length of $f$-faced bubbles. Each curve corresponds to a different time, as indicated by the legends. For clarity, each figure is split in 4, one for each different initial condition and a fourth one containing the scaling regime distributions reached from the three different initial configurations. 


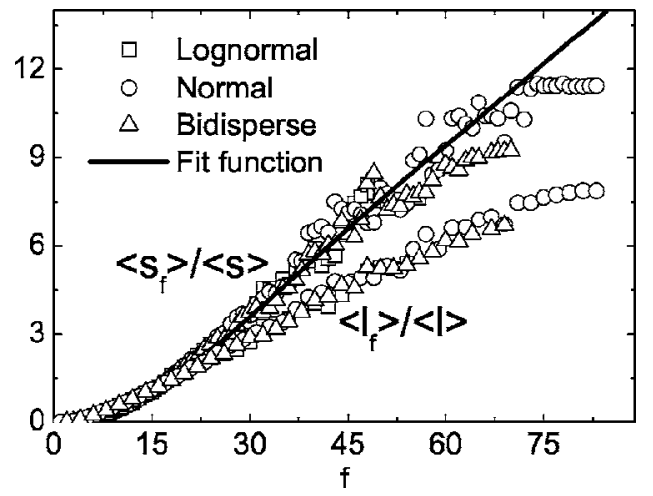

FIG. 17. $\langle s\rangle_{f} /\langle s\rangle$ and $\langle l\rangle_{f} /\langle l\rangle$ as functions of $f$, averaged over successive configurations in the scaling regime.

strongly depend on $f$, yielding a relation between $f$ and $\langle s\rangle_{f} /\langle s\rangle$ not very far from linear. Figure 17 shows that this is true for $f>15$ (the deviation for $f>50$ is probably not significant, given the small number of bubbles concerned). In Fig. 17 we also show a fit to $\langle s\rangle_{f} /\langle s\rangle$, where we have averaged over the simulation data up to $f=45$. We have extrapolated to $f>45$ using a straight line, to be used as input data for the theoretical model presented in Sec. IV, consistent with the assumption of uniform face areas for large $f$.

Figure 18 presents $\langle v\rangle_{f} /\langle v\rangle$ averaged over scaling regime configurations, as explained above, together with theoretical predictions. Consistently with the fit for $\langle s\rangle_{f} /\langle s\rangle$ used as input to the model, the deviation from the experimental data happens for $f>45$. In the inset, we present a log-log plot of the same data. Both a fit using a $f^{9 / 4}$ power law, and the theory (which does not go as $f^{9 / 4}$ ) describe well the simulation data for $f<55$. This data is in good agreement with Surface Evolver simulation data by Kraynik and collaborators [33] for random foams.

Figure 19 shows the scaling average of the probability of $f$-faced cells. Theoretical predictions agree well with simulation data. The deviations for small $f$ are probably due to

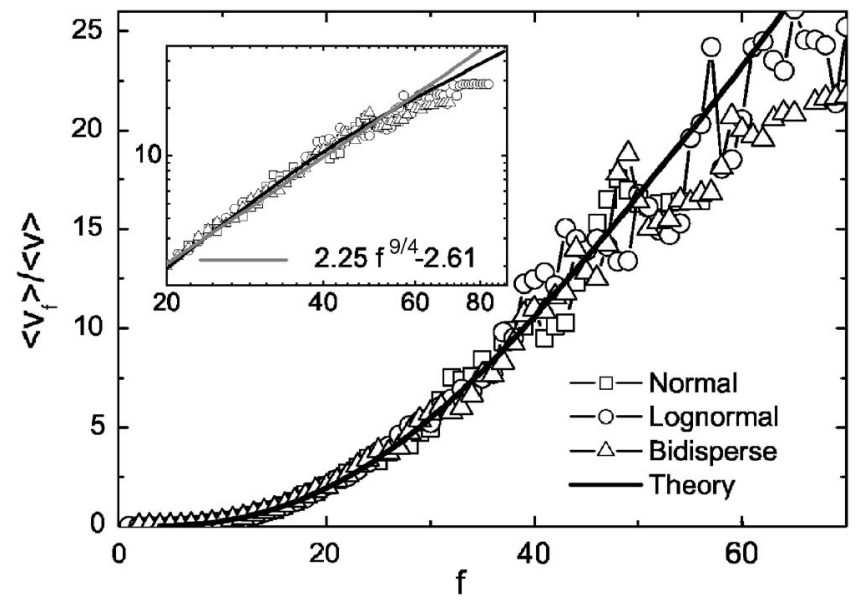

FIG. 18. $\langle v\rangle_{f} /\langle v\rangle$ as function of $f$ averaged over successive configurations in the scaling regime, together with theoretical predictions. In the inset we present the log-log plot, including a power law $f^{9 / 4}$.

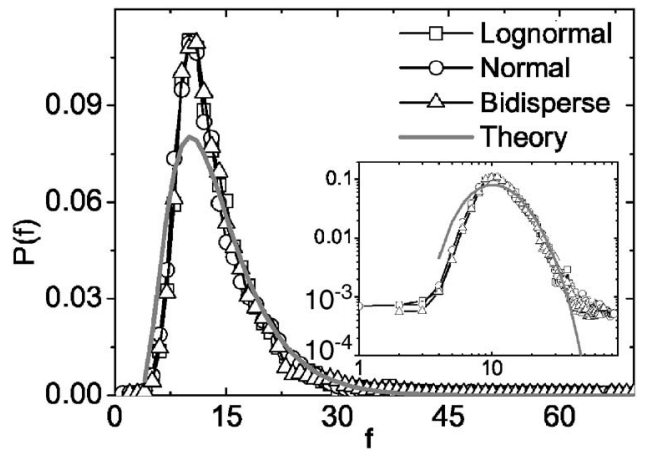

FIG. 19. $P(f)$ averaged over successive configurations in the scaling regime, together with the theoretical prediction. In the inset we present the log-log plot.

lattice effects that are more important for cells with few faces. Observe that the long time distribution presents a long tail and is not peaked at the average face number 13.7, but rather around 10.5, consistently with a high value of $\mu_{2}$.

Another dimensionless characterization is the topology of the faces themselves: a bubble may present faces with different numbers of edges. We have thus monitored the spread in the number of edges of the faces of a given bubble. For each bubble, we calculate the average number of edges per face epf and the standard deviation $\sigma_{e p f}$ of the distribution of edges for the cell. We then may obtain the distribution in the average number of edges and the average standard deviation, presented in Figs. 20.

The average number of edges per bubble for $f$-faced bubbles is fixed by the Euler theorem [5]), and is thus a straight line [Fig. 21(a)]. Conversely, the standard deviation of number of edges per bubble [Fig. 21(b)] is not constrained, and tends to saturate at large $f$; it does not depend much on time, even at the beginning.

\section{Evolution of correlations}

Since we are dealing with arbitrary units for surface tension and MCSs (assimilated to the time, for the sake of simplicity), we define the growth rate of $f$-faced bubbles up to a prefactor as

$$
G(f) \propto\left\langle v^{-1 / 3}(d v / d t)\right\rangle_{f},
$$

where $d t=1$ MCS. We perform the average over the $f$-faced bubbles that do not undergo a change in their number of neighbors during this MCS.

We present in Fig. 22 the plot of the average growth rate of $f$-faced cells for different times for each run with different initial conditions and a fourth plot with the scaling configurations for the different runs. Again all runs indicate a convergence to a unique scaling state. However, prior to the scaling regime, the growth rate may present different shapes. There is a minimum in the growth rate for low $f$-faced bubbles. In the scaling regime this minimum happens exactly for $f=4$, but before scaling this minimum may happen for $f=5$ or 3 . Bubbles with $f<4$ are not in equilibrium, do not obey Plateau's rules, and are absent in the scaling regime, in agreement with the right lower plot in Fig. 22. 

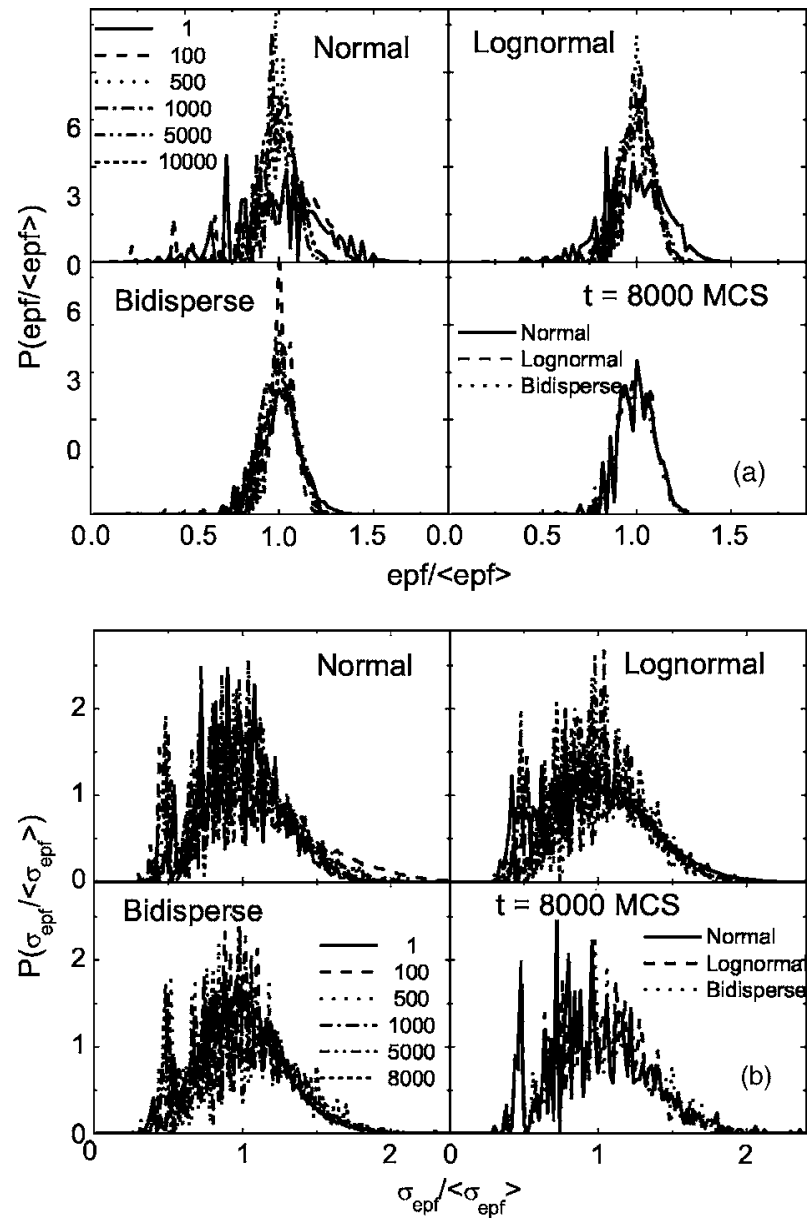

FIG. 20. Distributions of relative number of edges per face, and its relative standard deviation. For clarity, each figure is split in 4, one for each different initial condition and a fourth one containing the scaling regime distributions reached from the three different initial configurations.

In Fig. 23 we present growth rate scaling averages for the three different initial conditions. To estimate the spread of growth rates at the scaling regime, we have also plotted points representing all individual bubbles for $T=8000 \mathrm{MCSs}$ for the three initial conditions. The inset shows a zoom around $f_{0}$, the point at which the growth rate scaling averages cross the abscissa axis. We observe that $16<f_{0}<17$, close to our value of $\left\langle f^{2}\right\rangle /\langle f\rangle=\left(\mu_{2}+\langle f\rangle^{2}\right) /\langle f\rangle=16.4$. This is reminiscent of Glazier's suggestion that $f_{0}=\left\langle f^{2}\right\rangle /\langle f\rangle$ [20], even though our simulations do not obey his assumption that $\langle v\rangle_{f}$ goes as $f^{3}$.

We also address the possibility of the growth rate depending on volume rather than on number of faces only. In Fig. 24 we plot the growth rate of $f$-faced bubbles as a function of $v /\langle v\rangle_{f}$ for different values of $f$. These data are taken from a large simulation with initially bidisperse volume distribution, already in the scaling state. The growth rates are averaged taking the system configuration every $500 \mathrm{MCSs}$ from $t$ $=6000 \mathrm{MCSs}$ to $t=9500 \mathrm{MCSs}$. Figure 24 shows that there is not a marked tendency, indicating a volume independent growth rate for fixed values of $f$.

Finally, we present in Fig. 25 the first neighbor correlation in number of faces, that is the $3 \mathrm{D}$ equivalent of Aboav-
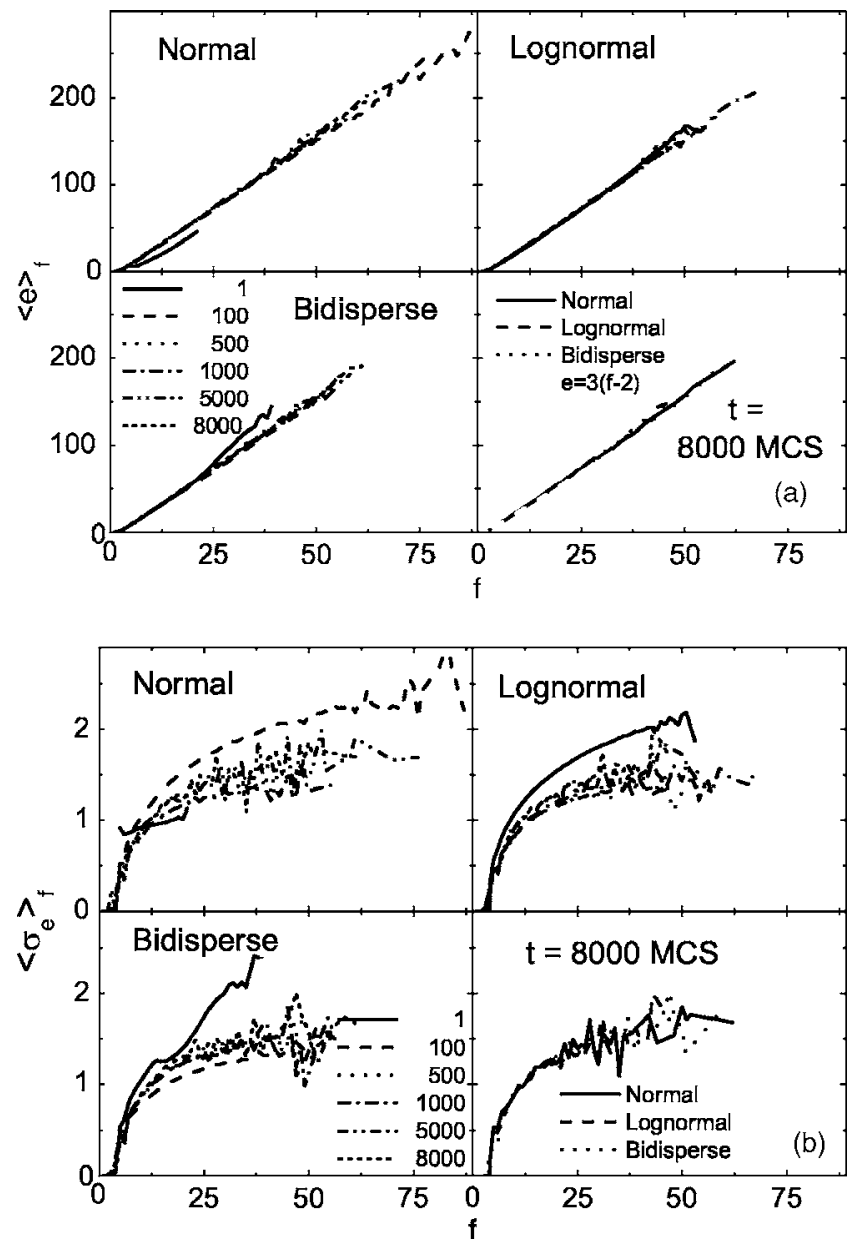

FIG. 21. Relative average number $\langle e\rangle_{f}$ (a) and its standard deviation $\left\langle\sigma_{e}\right\rangle_{f}$ (b) of edges per bubble of $f$-faced bubbles. Each figure is split in 4 , one for each different initial condition and a fourth one containing the scaling regime distributions reached from the three different initial configurations. The lower, right hand side plot in (a) also shows the Euler relation ([5]), $e=3 f-6$ as a gray dashed line.

Weaire law, here given as the plot of the scaling average of $f\langle m\rangle_{f}$ vs $f$, where $\langle m\rangle_{f}$ is the average number of faces of neighbors of a $f$-faced cell. In the same figure we have also plotted $f\langle m\rangle_{f}$ vs $f$, where $\langle m\rangle_{f}$ for individual bubbles of normal, lognormal, and bidisperse runs at 8000 MCSs. A linear fit of the averages is given as $f\langle m\rangle_{f}=14.47 f+26.45$. The agreement is almost perfect.

Following Jurine et al. [15] we check whether we can generalize the 2D formula proposed by Weaire [37] for the first neighbor correlation in number of faces:

$$
f\langle m\rangle_{f}=(\langle f\rangle-a) f+\left(a\langle f\rangle+\mu_{2}\right),
$$

where $a$ is a parameter. To independently estimate $a$ we have used the formula proposed by Kraynik [38]:

$$
a=\langle f\rangle-\frac{\left\langle f^{2}\langle m\rangle_{f}\right\rangle-\langle f\rangle\left\langle f^{2}\right\rangle}{\mu_{2}}
$$

in the following configurations: lognormal, $T=3500,4000$, and $4500 \mathrm{MCSs}$, and finally bidisperse, $T=6500$, 8000, and 


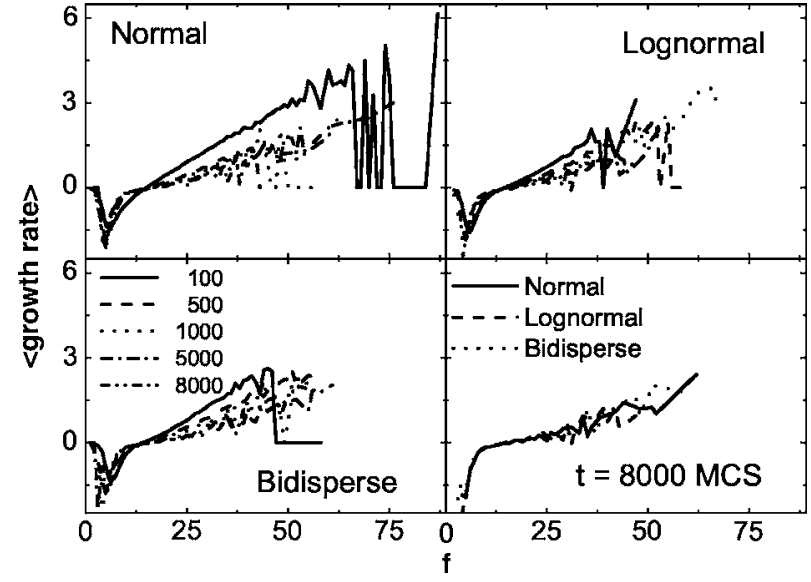

FIG. 22. Average growth rate of $f$-faced bubbles [Eq. (6)] at different instants, in arbitrary units. Each figure is split in 4, one for each different initial condition and a fourth one containing the scaling regime distributions reached from the three different initial configurations.

9500 MCSs. They all produced values of $a$ varying from -0.93 to -0.73 , yielding angular and linear coefficients for the linear plots, respectively, in the intervals $(14.44,14.71)$ and $(24.35,27.15)$. These linear plots are shown in the inset of Fig. 25, together with the scaling averages.

\section{THEORY}

\section{A. Model}

\section{Motivations}

The preceding section shows that a foam which has coarsened long enough attains a universal regime regardless of its initial condition. In what follows, we assume that it is fully disordered, that is, it maximizes its entropy within the constraints due to geometry and topology.

In two dimensions, the maximum entropy hypothesis led to strict predictions regarding topological distributions, in

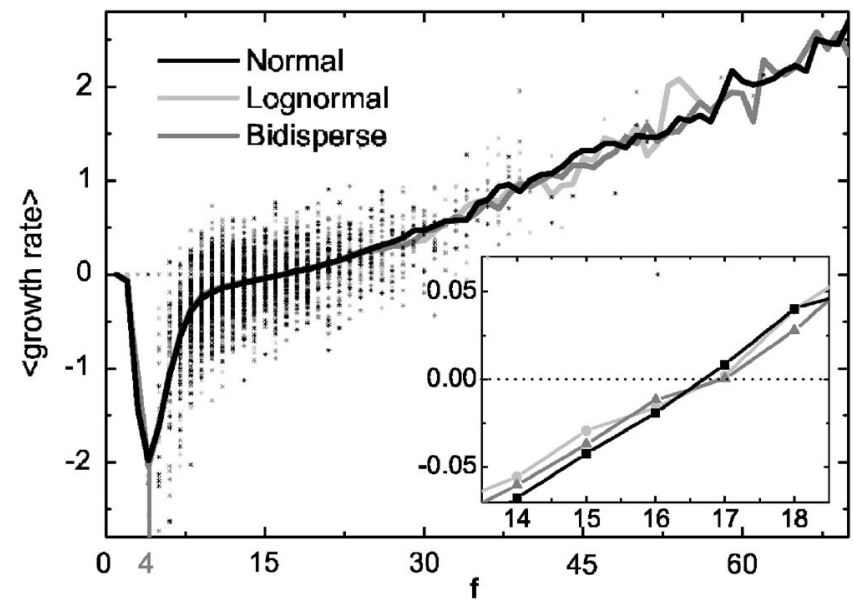

FIG. 23. Growth rate scaling averages of $f$-faced bubbles for three different initial conditions (solid lines) in arbitrary units and the plot of growth rate for all bubbles at $T=8000$ MCSs for each run. The inset shows a zoom around $f_{0}$ for the scaling averages.

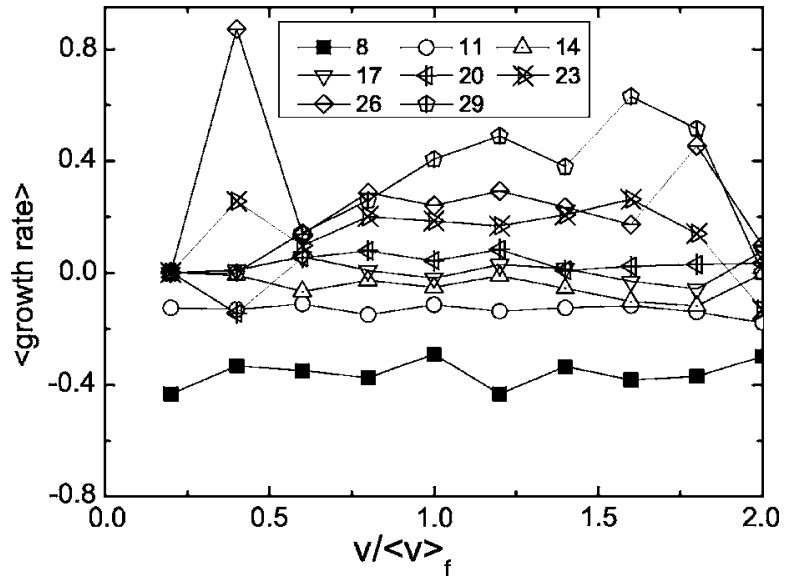

FIG. 24. Growth rate scaling averages as function of $v /\langle v\rangle_{f}$ for different values of $f$. Larger fluctuations happen for lower probability of $f$.

good agreement with well known experimental observations, such as the 2D Aboav-Weaire law [39-44].

Since the structure of foams is determined by energy minimization $[3,5,34]$, it is natural to refine this approach by including energy as well as entropy [45]. This is in fact a requirement if we want to determine not only the topological but also the geometrical distributions. This task had been performed by de Almeida et al., in two dimensions [46], then in three dimensions [47]. The results agreed well with the available data from $2 \mathrm{D}[48,49]$ and $3 \mathrm{D}$ experiments and simulations [12].

Accounting for both energy minimization and entropy maximization means minimizing free energy. Competition between energy and entropy is tuned by one free parameter, formally an effective temperature. This parameter, noted $T_{\text {eff }}$, is a free parameter not physically related to room temperature (see Sec. V B) in the sense that it is related to the aver-

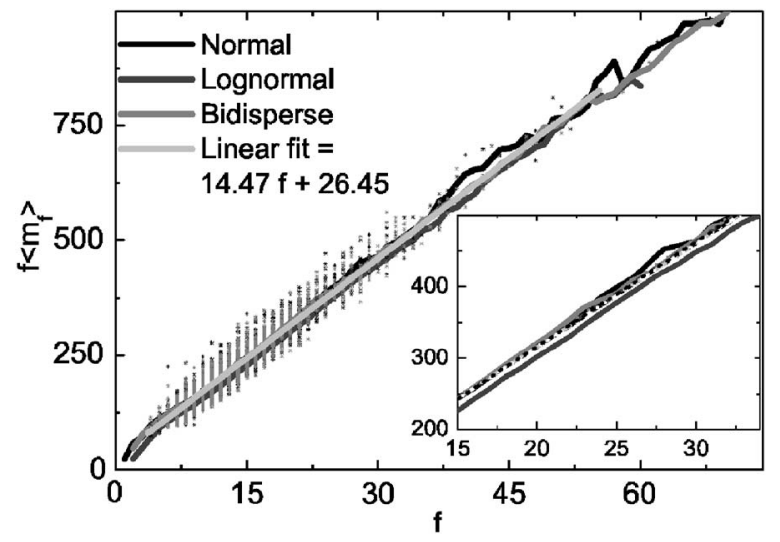

FIG. 25. Correlation between the number of faces of a cell and of its neighbors in the scaling regime. Solid lines: scaling averages for normal, lognormal, and bidisperse initial conditions, and for a linear fit. Asterisks: individual bubbles at $T=8000 \mathrm{MCSs}$ for normal, lognormal, and bidisperse. Inset presents solid lines as scaling averages for normal, lognormal, and bidisperse, and other line styles represent fits using $a$ as calculated by Eq. (8) for different configurations in the scaling regime (see text). 
age energy of the bubbles instead to their molecular components. If we also add an assumption regarding size-topology correlation, we have enough constraints to fully determine the structure of the foam.

Here, we refine de Almeida et al.'s theory to compare with simulations. Our main improvement is that the simulation results provide the theoretical model with a precise correlation between $\langle s\rangle_{f}$ and $f$ (that is, how the surface area of the bubbles increases with their number of faces), making an arbitrary guess unneeded.

\section{Ingredients of the model}

We start by considering a collection of $N$ bubbles, which are characterized by their number of faces $f$, surface area $s$ and volume $v$. Although volume, surface area, and number of faces are independent variables, they must respect a nonholonomic constraint which can be expressed by the maximum volume $v_{\max }(f, s)$ that a $f$-faced bubble of surface area $s$ may present, which is given by

$$
v_{\max }(f, s)=k(f) s^{3 / 2},
$$

where $k(f)$ is a form factor, that depends on the number of faces of the bubble.

As bubbles in a soap froth are supposed to obey Plateau rules, in this model we obtain $k(f)$ from isotropic Plateau polyhedra (IPP) which minimizes surface for a given volume subject to the constraint of obeying Plateau rules. In this case, Hilgenfeldt et al. [13] estimated the ratio $\beta$ $=s /\left(36 \pi v^{2}\right)^{1 / 3}$ as

$$
\beta(f) \sim 1.0813+\frac{0.01743}{f^{1 / 2}}+\frac{0.00228}{f}
$$

yielding a form factor $k(f)$ as

$$
k(f)=\frac{1}{\sqrt{36 \pi \beta^{3}}} .
$$

Since geometry, and not only topology, plays an essential role in the froth structure and evolution, we have chosen to minimize a free energy, rather than a pure entropy $([42,43])$. This choice is based on at least two reasons.

The first reason is purely geometrical. Neighboring bubbles (even with different number of faces) share a face, as well as its corresponding edges. This trivial correlation between neighbors induces nontrivial constraints on the possible functions that might describe the average surface area of $f$-faced bubbles.

The second reason regards energy, which drives the foam evolution, and is fully correlated with geometry. By definition, surface energy is proportional to the surface area of the bubbles, the prefactor being the surface tension $\sigma$ of a wall (i.e., two air-water interfaces in the case of soap films):

$$
E=\frac{\sigma}{2} \sum_{i=1}^{N} s_{i}
$$

Simulations indicate that the asymptotic regime is statistically invariant under transformations due to the coarsening.
That is, all dimensionless quantities are left invariant under both basic topological processes: "T1" neighbor swapping and "T2" bubble disappearance. In the calculation that follows, we thus use an equilibrium statistical mechanics formalism.

We use a single bubble approximation that allows computing the partition function. This is equivalent to assuming the bubbles do not interact: this is an "ideal foam" model. As a consequence, in order to make it possible to build a connected froth, and not just a collection of loose bubbles, we must introduce some correlation between bubbles [Eq. (16)] $[42,43]$. In two dimensions, it is natural to use the correlation between bubble number of sides and side size (the so-called empirical "Feltham law" [46,50]. In three dimensions, there are two possible choices, the correlation between $f$ and either $s$ [Fig. 16(c)] or $l$ [Fig. 16(d)]. Both are acceptable, especially since simulations show that both are independent of time, even well before reaching the asymptotic state [Figs. 16(c) and 16(d)] moreover, assuming one of these relations will lead to predicting the other [47]. We choose $s$ because it makes the calculation much simpler [47], since we can hence disregard edge length as a relevant variable of the model, that is, a variable that enters in the dynamical equations.

Euler relation enforces another relation between the average numbers of edges, of faces, and of vertices $[5,6]$, but leaves free one parameter, for instance $\langle f\rangle$. We include in the model the data from our simulation, that is $\langle f\rangle=13.7$ in the asymptotic regime (Fig. 7). We thus eliminate the number of edges in what follows.

\section{Basic equations}

The probability density function of a froth of $N$ bubbles is defined as a function $\rho_{N}$ of all variables of the system, that is,

$\rho_{N}=\rho_{N}\left(f_{1}, f_{2}, \ldots, f_{N} ; s_{1}, s_{2}, \ldots, s_{N} ; v_{1}, v_{2}, \ldots, v_{N} ; \vec{r}_{1}, \vec{r}_{2}, \ldots \overrightarrow{r_{n}}\right)$,

where $f_{i}, s_{i}, v_{i}$, and $\vec{r}_{i}$ are, respectively, the number of faces, surface area, volume, and center of mass position of the $i$ th bubble.

We define the following sum operator over the phase space:

$$
\begin{aligned}
\Phi= & \int_{V} d \overrightarrow{r_{1}} \cdots \int_{V} d \overrightarrow{r_{N}} \sum_{f_{1}=f^{*}}^{\infty} \cdots \sum_{f_{N}=f^{*}}^{\infty} \int_{0}^{\infty} d s_{1} \\
& \cdots \int_{0}^{\infty} d s_{N} \int_{0}^{v_{1}^{\max }} d v_{1} \cdots \int_{0}^{v_{N}^{\max }} d v_{N},
\end{aligned}
$$

where $v_{i}^{\max }=k\left(f_{i}\right) s_{i}^{3 / 2}$ is the maximum volume for a $f_{i}$-faced bubble of surface area $s_{i}$, and $f^{*}$ is the minimum number of faces of a bubble. Assuming that the bubbles are undistinguishable objects, the entropy is defined as

$$
S=-k_{B} \Phi \rho \ln \left(\frac{\lambda^{N} \rho}{N !}\right),
$$

where $\lambda$ has units of [length $]^{8}$, that is, a power 2 for surface, 3 for volume, and 3 for the center of mass position of each bubble. 
The probability density $\rho$ should maximize entropy subjected to the following constraints:

$$
\begin{gathered}
\Phi \rho=1, \\
\Phi \rho \sum_{i=1}^{N} \frac{\sigma}{2} s_{i}=\langle E\rangle, \\
\Phi \rho \sum_{i=1}^{N} f_{i}=2 F, \\
\Phi \rho \sum_{i=1}^{N} \delta\left(f_{i}-f\right)\left[s_{i}-\langle s\rangle_{f}\right]=0 .
\end{gathered}
$$

Here the first constraint is related to normalization. The second expresses the energy $E$ of the system. The third fixes the total number of faces in the froth as $F$, hence fixing the average number of faces of a bubble as $\langle f\rangle=2 F / N$. Finally, the last equation fixes the average surface area of $f$-faced bubbles to an input function $\langle s\rangle_{f} /\langle s\rangle$, that should be taken from simulation or experimental data, as discussed above.

In order to find the probability density function that respects the constraints represented by Eqs. (16), we must build the thermodynamical potential $\Psi$ which we have to maximize. It is

$$
\begin{aligned}
\Psi= & -k_{B} \Phi \rho \ln \left(\frac{\lambda^{N} \rho}{N !}\right)-\eta_{0} \Phi \rho-\eta_{1} \Phi \rho \sum_{i=1}^{N} \frac{\sigma}{2} s_{i}-\eta_{2} \Phi \rho \sum_{i=1}^{N} f_{i} \\
& -\sum_{f}^{\infty} \eta_{3}(f) \Phi \rho \sum_{i=1}^{N} \delta\left(f_{i}-f\right)\left[s_{i}-\langle s\rangle_{f}\right],
\end{aligned}
$$

where the $\eta_{i}$ 's are Lagrange multipliers associated to the constraint equations. The resulting probability density function is

$$
\begin{aligned}
\rho= & \left(\frac{V}{N \lambda}\right)^{N} \exp \left(-1-\frac{\eta_{0}}{k_{B}}\right) \times \exp \left(-\frac{\eta_{1}}{k_{B}} \frac{\sigma}{2} \sum_{i=1}^{N} s_{i}\right) \\
& \times \exp \left(-\frac{\eta_{2}}{k_{B}} \sum_{i=1}^{N} f_{i}\right) \times \exp \left(-\sum_{i=1}^{N} \frac{\eta_{3}\left(f_{i}\right)}{k_{B}}\left(s_{i}-\langle s\rangle_{f_{i}}\right)\right)
\end{aligned}
$$

with the restriction that $v_{i} \leqslant k\left(f_{i}\right) s_{i}^{3 / 2}$. Here we have assumed that the integrals over the center of mass positions is $V$ and also that $N ! \propto N^{N}$. The values of the Lagrange multipliers are determined through the constraint equations (16).

In what follows we simplify the form of the equations by introducing the simpler notation:

$$
\alpha_{i}=\frac{\eta_{i}}{k_{B}}
$$

As usual, $\alpha_{0}$ is associated to the partition function $Z$, which is given by

$$
\exp \left(1+\alpha_{0}\right)=Z=\left(\frac{Q V}{\lambda N}\right)^{N},
$$

where $Q$ is a reduced partition function, defined as follows:

$$
\begin{aligned}
Q= & \sum_{f}^{\infty} \int_{0}^{\infty} d s \int_{0}^{v_{\max }(f, s)} d v \\
& \times \exp \left(-\frac{\alpha_{1} \sigma}{2} s-\alpha_{2} f-\alpha_{3}(f)\left(s-\langle s\rangle_{f}\right)\right) \\
= & \frac{3 \sqrt{\pi}}{4} \sum_{f}^{\infty}\left[\frac{k(f)}{\left[\frac{\alpha_{1} \sigma}{2}+\alpha_{3}(f)\right]^{5 / 2}} \exp \left[-\alpha_{2} f+\alpha_{3}(f)\langle s\rangle_{f}\right] .\right.
\end{aligned}
$$

\section{B. Theoretical results}

\section{Resolution of the equations}

Since $N=\Sigma_{f}^{\infty} N_{f}$, where $N_{f}$ is the number of $f$-faced bubbles, from Eq. (21) we can deduce that the probability $P(f)$ of a $f$-faced bubble is

$$
P(f)=\frac{3 \sqrt{\pi}}{4 Q}\left[\frac{k(f)}{\left.\frac{\alpha_{1} \sigma}{2}+\alpha_{3}(f)\right]^{5 / 2}} \exp \left[-\alpha_{2} f+\alpha_{3}(f)\langle s\rangle_{f}\right] .\right.
$$

We can also calculate the average surface area of the bubbles:

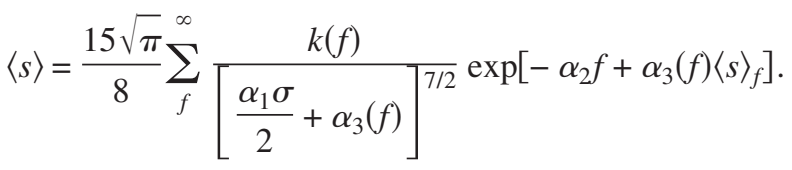

Knowing that $\langle s\rangle=\sum_{f}^{\infty} P(f)\langle s\rangle_{f}$, and comparing Eqs. (22) and (23), we can determine $\alpha_{3}(f)$ :

$$
\alpha_{3}(f)=\frac{5}{2\langle s\rangle_{f}}-\frac{\alpha_{1} \sigma}{2} .
$$

That leaves us with two parameters to determine: $\alpha_{1}$ and $\alpha_{2}$. We do it by numerically solving the following equations:

$$
\begin{aligned}
& \sum_{f}^{\infty} k(f)\left(\frac{\langle s\rangle_{f}}{\langle s\rangle}\right)^{5 / 2} \exp \left(-\alpha_{2} f-\frac{\alpha_{1} \sigma}{2}\langle s\rangle_{f}\right) \\
& =\sum_{f}^{\infty} k(f)\left(\frac{\langle s\rangle_{f}}{\langle s\rangle}\right)^{7 / 2} \exp \left(-\alpha_{2} f-\frac{\alpha_{1} \sigma}{2}\langle s\rangle_{f}\right), \\
& \langle f\rangle \sum_{f}^{\infty} k(f)\left(\frac{\langle s\rangle_{f}}{\langle s\rangle}\right)^{5 / 2} \exp \left(-\alpha_{2} f-\frac{\alpha_{1} \sigma}{2}\langle s\rangle_{f}\right) \\
& =\sum_{f}^{\infty} f k(f)\left(\frac{\langle s\rangle_{f}}{\langle s\rangle}\right)^{5 / 2} \exp \left(-\alpha_{2} f-\frac{\alpha_{1} \sigma}{2}\langle s\rangle_{f}\right) .
\end{aligned}
$$

At this point, to solve Eqs. (25), we introduce the correlation 


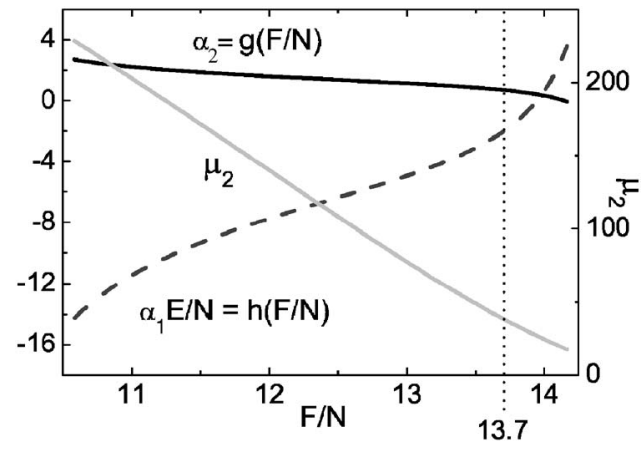

FIG. 26. Functions $g$ and $h$ vs $F / N=\langle f\rangle$, obtained as numerical solutions of Eqs. (25), from which $\alpha_{1}$ and $\alpha_{2}$ are determined. Also shown in the figure is the theoretical prediction for $\mu_{2}$.

between $\langle s\rangle_{f}$ and $f$. More precisely, since the equations use nondimensional quantities, we inject the relation between $f$ and $\langle s\rangle_{f} /\langle s\rangle$, obtained from a fit to the simulation results (Fig. 17), in the argument of the exponential function. We then solve numerically Eqs. (25) and obtain two monotonic functions of $F / N=\langle f\rangle$ (Fig. 26), namely

$$
\begin{gathered}
h\left(\frac{F}{N}\right)=\alpha_{1} \frac{\sigma\langle s\rangle}{2}, \\
g\left(\frac{F}{N}\right)=\alpha_{2} .
\end{gathered}
$$

Since, furthermore, $\sigma\langle s\rangle / 2=\langle E\rangle / N$, we can write $\alpha_{1}$ and $\alpha_{2}$ as functions of only the extensive variables $E, F$, and $N$. In principle, the problem is thus solved. The reduced density function $\rho_{r}$ is

$$
\begin{aligned}
\rho_{r}(f, s, v)= & \frac{\Theta\left[k(f) s^{3 / 2}-v\right]}{Q} \exp \left[\frac{5}{2}-g\left(\frac{F}{N}\right) f-h\left(\frac{F}{N}\right) \frac{\langle s\rangle_{f}}{\langle s\rangle}\right. \\
& \left.-\frac{5 s}{2\langle s\rangle_{f}}\right],
\end{aligned}
$$

where $\Theta(x)$ is the Heaviside step function. We can now calculate the entropy as

$$
\begin{aligned}
S= & k_{B} \ln Z+k_{B} N h\left(\frac{F}{N}\right)+2 k_{B} F g\left(\frac{F}{N}\right)=N k_{B} \ln \left(\frac{V}{N}\right) \\
& +\frac{5 N}{2} k_{B} \ln \left(\frac{\langle E\rangle}{N \sigma / 2}\right)+k_{B} N \ln \left[\frac{3 \sqrt{\pi}}{4 \lambda}\left(\frac{2 e}{5}\right)^{5 / 2} S_{5 / 2}\right] \\
& +k_{B} N h\left(\frac{F}{N}\right)+2 k_{B} F g\left(\frac{F}{N}\right),
\end{aligned}
$$

where we have used the fact that $\langle E\rangle=\sigma\langle s\rangle N / 2$, and that the functions $h$ and $g$ are functions of $F / N$ only. Here $S_{5 / 2}$ is a dimensionless function and is defined as

$$
S_{5 / 2}=\sum_{f}^{\infty} \exp \left(-g f-h \frac{\langle s\rangle_{f}}{\langle s\rangle}\right) k(f)\left(\frac{\langle s\rangle_{f}}{\langle s\rangle}\right)^{5 / 2} .
$$

Observe that Eq. (28) has the same form as the fundamental equation of the diatomic ideal gas model in respect to the internal energy $\langle E\rangle$ and volume $V$. This is a direct consequence of having assumed only surface area energy (neglecting phase energy) and, of the implicit assumption present in taking the value of the center of mass integrals equal to $V$ when obtaining the form of the partition function $Z$ as given in Eq. (20).

Similarly, we also define $S_{4}$ as

$$
S_{4}=\sum_{f}^{\infty} \exp \left(-g f-h \frac{\langle s\rangle_{f}}{\langle s\rangle}\right) k^{2}(f)\left(\frac{\langle s\rangle_{f}}{\langle s\rangle}\right)^{4}
$$

and $S_{3}$ as

$$
S_{3}=\sum_{f}^{\infty} \exp \left(-g f-h \frac{\langle s\rangle_{f}}{\langle s\rangle}\right) k^{4 / 3}(f)\left(\frac{\langle s\rangle_{f}}{\langle s\rangle}\right)^{3} .
$$

\section{Predictions: Energy and geometry}

Now that we have determined all Lagrange multipliers, we can calculate quantities that may be compared with results obtained from experiments and/or simulations. Since these equations describe a scale invariant regime, distributions are expressed in dimensionless units which do not vary in time, while dimensional quantities as volume, for example, are given in units of average surface area $\langle s\rangle$ taken to the adequate power.

We start by calculating the average volume of the froth:

$$
\langle v\rangle=\frac{8 \sqrt{2}}{5 \sqrt{5 \pi}} \frac{S_{4}}{S_{5 / 2}}\langle s\rangle^{3 / 2},
$$

where $S_{5 / 2}$ and $S_{4}$ are given by Eqs. (29) and (30). The average value of the cubic root of volume, $\left\langle v^{1 / 3}\right\rangle$ is given by

$$
\left\langle v^{1 / 3}\right\rangle=\frac{2 \sqrt{2}}{\sqrt{5 \pi}} \frac{S_{3}}{S_{5 / 2}}\langle s\rangle^{1 / 2},
$$

where $S_{3}$ is given by Eq. (31).

We now discuss the effective temperature of the froth $T_{\text {eff }}$, which is defined as

$$
\frac{1}{T_{e f f}}=\left(\frac{\partial S}{\partial E}\right)_{N, F}=\frac{5}{2} \frac{N k_{B}}{\langle E\rangle} .
$$

This gives the following equation of state:

$$
\langle E\rangle=\frac{5}{2} N k_{B} T_{e f f},
$$

where the factor $5 / 2$ originates in the five linear dimensions of the phase space (surface area $\times$ volume) of a bubble [Eq. (15)]. Such an equation of state corresponds to an "ideal foam," and simply defines $T_{\text {eff }}$ as the average energy per bubble.

This effective temperature is macroscopic and its order of magnitude is not related to a usual molecular kinetic energy. As an example, a usual soap froth with bubble diameter of, say, $1 \mathrm{~mm}$ and a surface energy of $10^{-2} \mathrm{~N} / \mathrm{m}$ has an energy per bubble of around $10^{-2} \times\left(10^{-3}\right)^{2}=10^{-8} \mathrm{~J}$, a small but certainly macroscopic value. The corresponding effective temperature is around $10^{15} \mathrm{~K}$, not related to the room tempera- 
ture. This is expected, since in a noninteracting system like the present ideal foam the temperature is not an independent parameter, and physically corresponds only to the energy. Observe, also, that as the model has been proposed, bubbles energy comes only from their surface, regardless of its volume. So, in practice, even $T_{\text {eff }}$ can be extracted from the simulations.

\section{Predictions: Topology distributions}

We now present the results for $f$-dependent quantities. We start by rewriting the probability of finding a $f$-faced bubble as

$$
P(f)=\frac{35 e^{5 / 2}}{25 Q} \sqrt{\frac{2 \pi}{5}}\langle s\rangle^{5 / 2} \exp \left(-g f-h \frac{\langle s\rangle_{f}}{\langle s\rangle}\right) k(f)\left(\frac{\langle s\rangle_{f}}{\langle s\rangle}\right)^{5 / 2},
$$

where $e=2.71828 \ldots$, and $Q$, the reduced partition function, is given by

$$
Q=\frac{3 e^{5 / 2}}{25} \sqrt{\frac{2 \pi}{5}}\langle s\rangle^{5 / 2} S_{5 / 2} .
$$

Figure 19 shows the plot of Eq. (36) for $\langle f\rangle=13.72$ and the corresponding value for $\mu_{2}=37.1$ (our numerical results are not limited in precision) together with the average of simulation data for successive times in the scaling regime for three different initial conditions. This value of $\langle f\rangle$ corresponds to $h=-1.85$ and $g=0.69$, which we shall take as the representative values for the scaling state. In what follows these are the values we shall use to compare with the scaling regime quantities we have obtained from the simulations. The agreement is very good, although the peak in the theoretical curve is a bit lower.

The relative average volume of $f$-faced bubbles is

$$
\frac{\langle v\rangle_{f}}{\langle v\rangle}=k(f)\left(\frac{\langle s\rangle_{f}}{\langle s\rangle}\right)^{3 / 2} \frac{S_{5 / 2}}{S_{4}},
$$

and in Fig. 18 we show the plot of the above equation together with simulations scaling regime relative volumes of $f$-faced bubbles. Finally, it is also possible to calculate the distributions function in the relative quantities as $v /\langle v\rangle$, $s /\langle s\rangle$, and $v^{1 / 3} /\left\langle v^{1 / 3}\right\rangle$ :

$$
\begin{aligned}
P_{v}\left(\frac{v}{\langle v\rangle}\right)= & \frac{16}{3 \pi} \frac{S_{4}}{S_{5 / 2}^{2}} \sum_{f}^{\infty} \frac{\langle s\rangle_{f}}{\langle s\rangle} \exp \left[-g f-h \frac{\langle s\rangle_{f}}{\langle s\rangle}\right. \\
& \left.-\frac{5}{2} \frac{\langle v\rangle^{2 / 3}}{k^{2 / 3}(f)\langle s\rangle_{f}}\left(\frac{v}{\langle v\rangle}\right)^{2 / 3}\right], \\
P_{s}\left(\frac{s}{\langle s\rangle}\right)= & \frac{25}{3} \sqrt{\frac{5}{2 \pi}} \frac{1}{S_{5 / 2}} \sum_{f}^{\infty} k(f)\left(\frac{s}{\langle s\rangle}\right)^{3 / 2} \exp \left(-g f-h \frac{\langle s\rangle_{f}}{\langle s\rangle}\right. \\
& \left.-\frac{5 s}{2\langle s\rangle_{f}}\right)
\end{aligned}
$$

and

$$
P_{v^{1 / 3}}\left(\frac{v^{1 / 3}}{\langle v\rangle^{1 / 3}}\right)=3 \frac{\left\langle v^{1 / 3}\right\rangle}{\langle v\rangle^{1 / 3}}\left(\frac{v}{\langle v\rangle}\right)^{2 / 3} P_{v}\left(\frac{v}{\langle v\rangle}\right) .
$$

In Fig. 13 we compare the theoretical prediction for the relative volume distribution with the simulation data for the scaling regime. This figure shows a good agreement between simulation data and theory for large volumes, presenting some deviations for intermediate and small volumes. In fact, theory predicts a higher relative number of very small bubbles. This characteristic is even more evident in Fig. 14 where we have plotted the theoretical prediction for the distribution of relative size $\left(v^{1 / 3} /\left\langle v^{1 / 3}\right\rangle\right)$ and relative surface area $(s /\langle s\rangle$. The conclusion is that the model predicts a higher dispersion in the size for the bubbles than that observed in the simulations.

In fact we have calculated the average values for all four polydispersity measures. Not surprisingly, $\mu_{2} /\langle f\rangle^{2}=0.197$ corresponds rather well to the simulation data: the model correctly predicts the value of $\mu_{2}$ for a given $\langle f\rangle$. However, other predictions for polydispersity measures are $p=0.33$ and $\sigma_{R} / R=0.62$ that significantly deviates from simulations results (compare with the values in Fig. 9 where $p=0.20$ and $\left.\sigma_{R} / R=0.45\right)$. Deviations for $\beta$ are even more dramatic. In fact, analytical calculations for the average values for $f$-faced bubbles yield

$$
\langle\beta\rangle_{f}=\frac{3}{\left[36 \pi k^{2}(f)\right]^{1 / 3}}=3 \beta(f),
$$

where $k(f)$ is the form factor defined in Eq. (11), and $\beta(f)$ refers to the isotropic Plateau polyhedra, corresponding to the solid line in Fig. 9 and given in Eq. (10). Theoretical predictions are hence three times higher than the lower limit for soap bubbles as predicted by Surface Evolver simulations [33].

This larger size dispersion predicted by the model is a consequence of the lack of an energy term that would prevent $f$-faced bubbles with a given surface area $s$ from presenting volumes much smaller than that of an IPP, corresponding to elongated anisotropic cells. This is prevented in real foams due to the gas phase energy, that resists to a decrease in volume due to the corresponding increase in the bubble internal pressure. A possible solution is to consider a volume energy term for the bubbles as considered in Ref. [47]. This may enhance polydispersity predictions but will be presented elsewhere.

\section{DISCUSSION}

The agreement between simulation and theory results reinforces the confidence on the two models. These two models, however, have different basic assumptions whose validity deserves further discussions: while simulation results stem from questions on the growth rate of individual bubbles, the theoretical model arbitrarily assumes ergodicity and specific energy terms. In the subsections that follow we discuss these items. 

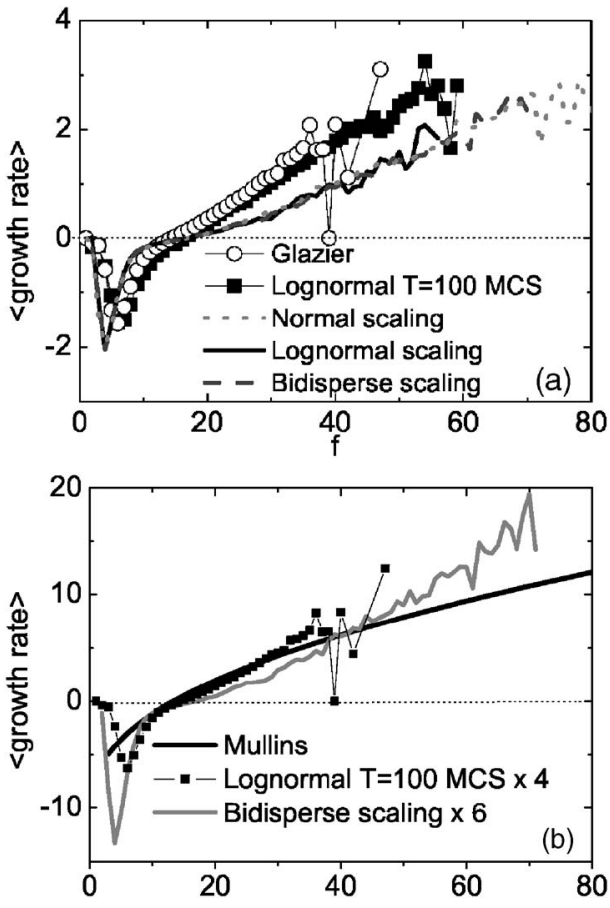

FIG. 27. Comparison between growth rates as obtained in Potts model simulations here and by Glazier [20], and analytical calculation by Mullins [10].

\section{A. Discussion of the simulations}

We begin by comparing the growth rate of individual $f$-faced bubbles (Fig. 27) with the data available in the literature, up to a prefactor. In Fig. 27(a) we present the plot of Potts model simulations by Glazier [20] (uncorrected data), together with our results for early times and for the scaling regime. This suggests that Glazier's simulations were not quite yet at the scaling regime. On the other hand, Mullins' law for the growth rate of regular 3D bubbles [10] displays a smoother curve, closer to a $G \sim \sqrt{f}$ tendency, that has been confirmed by analytical or numerical methods for regular and quasiregular bubbles, [11-16,51] (for reviews, see Refs. $[8,15]$ ). Figure 27 (b) shows our results both at an early time as well as at the scaling regime, rescaled by a multiplicative constant to fit Mullins curve. Mullins' predictions agree better with our early time results than with our scaling regime. It is important to understand the causes and consequences of this difference.

A first step is to compare the two different softwares that have been used to produce the curves: the Potts model on one hand, and Surface Evolver [35] for (quasi) regular bubbles on the other. It is thus natural to examine whether one model can have artifacts. Since Surface Evolver is very close to analytical results, the suspicion falls on the Potts model.

The Potts model has been widely used and validated on equilibrium or quasistatic simulations of foams. It is less precise than Surface Evolver for individual bubbles, but provides better statistics by allowing the manipulation of a larger number of bubbles. We emphasize that the discrepancy between different results is larger than the incertitude coming from discretization effects in surface area measurements or from statistical dispersion.

In fact, as discussed in Sec. II, there is an important difference between the Potts model and Surface Evolver which, in fact, also exists between grains and foams: surface tension is anisotropic in grains (as in Potts), while being isotropic in foams (as in models using Surface Evolver or the theoretical model presented here). Furthermore, together with discretization effects on the surface measure, this anisotropy leads to a peculiar surface area minimization that does not yield a sphere as the 3D figure with the least surface area for a given volume. If we are interested in foams rather than in grains, Surface Evolver is thus more adapted.

A more fundamental difference could originate in the fact that in 3D Mullins' law the growth rate of individual bubbles is not a function of $f$ only: although weakly, it does depend on the detailed properties of the bubbles being slightly different for ideally regular bubbles as compared to irregular real ones. In fact, there is a distribution of growth rates: a cloud of points and not a simple line (see Refs. [13-15] and Fig. 23).

Consequently, to obtain a realistic plot for the average growth rate of $f$-faced bubbles, it is necessary to have both a very large number of bubbles and a long coarsening time requirement which are both fulfilled in the present work. Moreover, as any other simulation of a whole disordered foam $[12,15]$, our simulations automatically satisfy the experimental constraint that the total volume is conserved during the coarsening. In analytical models this is a difficult point to check, although Mullins [10] finds a good agreement, using experimental distributions of the number of faces. On the other hand, when the growth rate is determined from instantaneous pressure differences on regular or quasiregular bubbles $[11,14,16,51]$, there is not a way to enforce or check total volume conservation.

Nevertheless, this whole discussion might become useless. In fact, the main question might be the following: If there was an artifact affecting the individual growth rate in the Potts model, how would it change the results presented here? Would they be meaningless, or just marginally affected? Rigorously answering this question would require to us simulate a foam, starting with a single initial condition, but coarsening with different enforced individual growth rates. Experiments with large bubble numbers [24] might also contribute the answer to this question in the future. However, the fact that different initial conditions tend towards the same state, that this state agrees with our model of a disordered foam, and that our results are compatible with the ones available on the literature, make us confident that there is no major surprise to expect.

\section{B. Discussion of the model}

We analytically model a disordered foam and verify that the results agree with those of the simulations of a scaling state. With respect to Refs. [46,47], we have considered fewer constraints. Especially, in the present version of the model we follow what happens in the Potts model simulations and do not include any volume energy term. We also 
use simulation results to feed the model with all parameter values, paying special attention to the geometrical correlation between variables: the average relative surface area of $f$-faced bubbles, $\langle s\rangle_{f} /\langle s\rangle$, the maximal volume $v_{\max }(s, f)$, and the minimal number of faces $f_{\min }$. Even the effective temperature $T_{\text {eff }}$ can finally be taken from the simulations, so that there is no free parameter left. These are a small conceptual change, but in practice they imply completely recalculating the distribution functions.

We also reinterpret the role played by Lagrange multipliers $\alpha_{1}$ and $\alpha_{2}$, giving a correct definition for effective temperature, allowing negative values for $\alpha_{1}$. This is so because, as opposed to usual statistical mechanics models, here temperature (as thermodynamically defined through the partial derivative of entropy in respect to energy) is not simply the inverse of the Lagrange multiplier associated to the energy constraint. From Eqs. (16) and (34) we can write

$$
T_{e f f}=\frac{2}{5 k_{B}} \frac{\sigma\langle s\rangle}{2},
$$

which, during the coarsening, is always increasing. Furthermore, observe that functions $h$ and $g$ [Eqs. (26)] are functions of $F / N$ only, and are fully determined by imposing a defined value for $\langle f\rangle$.

In fact, bubbles with different numbers of faces have different average surface area, and hence different average energy. The whole froth acts as a mixture of different kinds of particles, one kind for each number of faces. In this case, the exponent $h\langle s\rangle_{f} /\langle s\rangle$ plays the role in Eqs. (20) or (21) of the ratio of a chemical potential to the temperature. Chemical potentials can be negative, provided they do not cause any unphysical divergence.

We believe these modifications yield significant improvements. First we have one less parameter to deal with. Second, we can obtain higher values of $\mu_{2}$, consistent with simulation values, yielding more adequate distribution functions.

The present "ideal foam" model might be used as a sound basis over which we could design future refinements. These refinements should consider the introduction of a volume energy term, aiming at decreasing the probability of finding high $\beta$ cells, that is, bubbles with high surface area for a given volume and number of faces.

\section{CONCLUSION}

\section{A. Summary}

Our simulations establish a coherent picture, consistent with the literature, and with an analytical model of a disordered foam which accounts for energy, entropy, and a parameter tuning the balance between them. Starting from different initial conditions and coarsening during a long transient, foams or grains tend towards the same universal state. This state has a unique distribution of topological and geometrical quantities, as well as of their correlations. The distribution includes a significant number of bubbles up to 60 faces, and an almost linear correlation between the number of faces and edge lengths of the bubbles. In this state, bubbles with 16 faces or less shrink, bubbles with 17 faces or more tend to grow, resulting in stationary distributions and presenting, as expected, an overall increase in the average bubble size as $t^{1 / 2}$, due to bubble disappearances.

Such studies require enough bubbles in the initial foam, and enough CPU time, to satisfy three constraints: enough time for the transient to end and reach the scaling state, then enough time in the scaling state itself to characterize its properties, and enough bubbles at the end to avoid spurious finite size effects. In the case of the Potts model, we satisfy the three constraints simultaneously, if we manage to have around 5000 bubbles when the foam enters the asymptotic, statistically scale-invariant state (this is 450 times less than at the beginning). It is not clear whether this number will be smaller for other simulation techniques, or for experiments, which up to now still do not reach the scaling regime [24].

\section{B. Perspectives}

This work might open different perspectives. We do not study in detail the effect of initial conditions on the coarsening. Numerically, the transient should be as short as possible; even by starting from an already well-coarsened foam, the transient is just short enough for us to proceed. Starting from an unfavorable case (especially from an ordered state) could be untractable with our present tools. Analytically, we assume from the beginning that the system is already in the scaling state, and do not touch the point of how the distributions relax towards this state. Can we prove the scaling state exists and is reached whatever the initial conditions? This would require us to develop a formalism equivalent to nonequilibrium thermodynamics. An interesting alternative would be to extend the $2 \mathrm{D}$ master equation derived by Marder [52] to the 3D case.

It could also be interesting to see whether we can find in three-dimensions an equivalent of the 2D "Lemaitre law," which states that entropy minimization [42-44] predicts a strong correlation between the second moment $\mu_{2}(n)$ of the side number distribution, and the probability $P(6)$ of hexagons: for $0.34<P(6)<0.66, \quad \mu_{2}(n)+P(6)=1$; for 0.66 $<P(6)<1,2 \pi \mu_{2}(n) P(6)^{2}=1$.

In the future, we could perform experiments or simulations on moderately wet foams. We might determine whether there exists a scaling state for systems intermediate between the present scaling state, for completely dry foams, and the scaling state known in the other end, the completely wet bubbles (Ostwald ripening [53]). More generally, it would be interesting to replace the present study in the more general frame of coarsening systems [54].

\section{ACKNOWLEDGMENTS}

We are grateful to J. A. Glazier for the initial 3D Potts code. We thank J. A. Glazier, J. Lambert, I. Cantat, and R. Delannay for stimulating discussions. This work was partially supported by CAPES, COFECUB, and CNPq. 
[1] C. S. Smith, in Metal Interfaces (American Society for Metals, Cleveland, 1952), p. 65.

[2] J. von Neumann, in Metal Interfaces, (Ref. [1]), pp. 108-110, quoted, e.g., in Refs. [4,6,7].

[3] D. Weaire and N. Rivier, Contemp. Phys. 25, 59 (1984).

[4] J. A. Glazier and D. Weaire, J. Phys.: Condens. Matter 4, 1867 (1992).

[5] D. Weaire and S. Hutzler, The Physics of Foams (Clarendon Press, Oxford, 1999).

[6] J. A. Glazier, Ph.D. thesis, University of Chicago, Chicago, 1989.

[7] J. Stavans, Rep. Prog. Phys. 56, 733 (1993).

[8] J. Glazier and B. Prause, in Foams, Emulsions and Their Applications, edited by P. Zitha, J. Banhart, and G. Verbist (MITVerlag, Bremen, 2000), pp. 120-127.

[9] D. Weaire and J. Glazier, Philos. Mag. Lett. 68, 363 (1993).

[10] W. W. Mullins, Acta Metall. 37, 2979 (1989).

[11] J. E. Avron and D. Levine, Phys. Rev. Lett. 69, 208 (1992).

[12] F. Wakai, N. Enomoto, and H. Ogawa, Acta Mater. 48, 1297 (2000)

[13] S. Hilgenfeldt, A. Kraynik, D. Reinelt, and J. Sullivan, Europhys. Lett. 67, 484 (2004).

[14] S. J. Cox and F. Graner, Phys. Rev. E 69, 031409 (2004).

[15] S. Jurine, S. Cox, and F. Graner, Colloids Surf., A 263, 18 (2005); Proceedings of the 5th European Conference on Foams, Emulsions, and Applications, EUFOAM 2004, Marne La Vallée, 5-8 July 2004, edited by M. Vignes-Adler, D. Weaire, and R. Miller.

[16] S. Hilgenfeldt, A. M. Kraynik, S. A. Koehler, and H. A. Stone, Phys. Rev. Lett. 86, 2685 (2001).

[17] A. Kraynik, D. Reinelt, and F. van Swol, Colloids Surf., A 263, 11 (2005); Proceedings of the 5th European Conference on Foams, Emulsions, and Applications, EUFOAM 2004 (Ref. [15]).

[18] A. Kraynik, S. Hilgenfeldt, and D. Weaire (unpublished).

[19] W. M. Mullins, J. Appl. Phys. 59, 1341 (1986).

[20] J. A. Glazier, Phys. Rev. Lett. 70, 2170 (1993).

[21] C. P. Gonatas, J. S. Leigh, A. G. Yodh, J. A. Glazier, and B. Prause, Phys. Rev. Lett. 75, 573 (1995).

[22] C. Monnereau and M. Vignes-Adler, Phys. Rev. Lett. 80, 5228 (1998).

[23] B. Prause and J. A. Glazier, Proceedings of the 5th Experimental Chaos Conference, edited by M. Ding, W. L. Ditto, L. M. Pecora, and M. L. Spano (World Scientific, Singapore, 2001), p. 427.

[24] J. Lambert, I. Cantat, R. Delannay, A. Renault, F. Graner, J. A. Glazier, I. Veretennikov, and P. Cloetens, Colloids Surf., A 263, 295 (2005); Proceedings of the 5th European Conference on Foams, Emulsions, and Applications, EUFOAM 2004 (Ref. [15]).

[25] M. P. Anderson, G. S. Grest, and D. J. Srolovitz, Philos. Mag. B 59, 293 (1989).
[26] K. Fuchizaki, T. Kusaba, and K. Kawasaki, Philos. Mag. B 71, 333 (1995); K. Fuchizaki and K. Kawasaki, Physica A 221, 202 (1995).

[27] J. Ashkin and E. Teller, Phys. Rev. 64, 178 (1943).

[28] R. B. Potts, Proc. Cambridge Philos. Soc. 48, 106 (1952).

[29] F. Y. Wu, Rev. Mod. Phys. 54, 235 (1982).

[30] E. A. Holm, J. A. Glazier, D. J. Srolovitz, and G. S. Grest, Phys. Rev. A 43, 2662 (1991).

[31] Y. Jiang, J.-C. M. Mombach, and J. A. Glazier, Phys. Rev. E 52, R3333 (1995).

[32] Morphology of Condensed Matter-Physics and Geometry of Spatially Complex Systems, edited by K. Mecke and D. Stoyan, Lecture Notes in Physics No. 600, (Springer, Heidelberg, 2002).

[33] A. M. Kraynik, D. A. Reinelt, and F. van Swol, Phys. Rev. Lett. 93, 208301 (2004).

[34] F. Graner, Y. Jiang, E. Janiaud, and C. Flament, Phys. Rev. E 63, 011402 (2001).

[35] K. Brakke, Exp. Math. 1, 141 (1992).

[36] V. Fradkov, L. Schvindlerman, and D. Udler, Scr. Metall. 19, 1285 (1985).

[37] D. Weaire, Metallography 7, 157 (1974).

[38] A. Kraynik (private communication); quoted in Ref [15].

[39] F. Elias, C. Flament, J. A. Glazier, F. Graner, and Y. Jiang, Philos. Mag. B 79, 729 (1999).

[40] D. Weaire, S. J. Cox, F. Graner, Eur. Phys. J. E 7, 123 (2002).

[41] R. Höhler, S. Cohen-Addad, and A. Asnacios, Europhys. Lett. 48, 93 (1999).

[42] N. Rivier, in Foams and Emulsions, edited by J. J. Sadoc and N. Rivier (Kluwer, Boston, 1999), pp. 105-125.

[43] N. Rivier, in Disorder and Granular Media, edited by D. Bideau and A. Hansen (Elsevier, New York, 1993), pp. 55102.

[44] J. Lemaître, A. Gervois, J. P. Troadec, N. Rivier, M. Ammi, L. Oger, and D. Bideau, Philos. Mag. B 67, 347 (1993).

[45] G. Schliecker and S. Klapp, Europhys. Lett. 48, 122 (1999).

[46] R. M. C. de Almeida, and J. R. Iglesias, J. Phys. A 21, 3365 (1988); J. R. Iglesias and R. M. C. de Almeida, Phys. Rev. A 43, 2763 (1991).

[47] R. M. C. de Almeida and J. C. M. Mombach, Physica A 236, 268 (1997).

[48] J. A. Glazier and J. Stavans, Phys. Rev. A 40, 7398 (1989).

[49] J. Stavans and J. A. Glazier, Phys. Rev. Lett. 62, 1318 (1989).

[50] K. Y. Szeto and W. Y. Tam, Physica A 221, 256 (1995).

[51] S. Cox and M. Fortes, Philos. Mag. Lett. 83, 281 (2003).

[52] M. Marder, Phys. Rev. A 36, 438 (1987).

[53] W. Ostwald, Grundriss der Allgem. Chemie (Macmillan, London, 1908), p. 96; Foundations of Analytic Chemistry, 3rd English edition (Macmillan, London, 1908), p. 22; Principles of Inorganic Chemistry (Macmillan, London, 1902), p. 258.

[54] M. Seul, N. Y. Morgan, and C. Sire, Phys. Rev. Lett. 73, 2284 (1994). 